\title{
Review \\ Optical Spectrometry to Determine Nutrient Concentrations and other Physicochemical Parameters in Liquid Organic Manures: A Review
}

\author{
Michael Horf ${ }^{1, *(\mathbb{C}}$, Sebastian Vogel ${ }^{1}$, Harm Drücker ${ }^{2}$, Robin Gebbers ${ }^{1}{ }^{(D)}$ and Hans-Werner Olfs ${ }^{3}(\mathbb{D})$ \\ 1 Department Engineering for Crop Production, Leibniz Institute for Agricultural Engineering and \\ Bioeconomy (ATB), Max-Eyth-Allee 100, 14469 Potsdam, Germany; svogel@atb-potsdam.de (S.V.); \\ rgebbers@atb-potsdam.de (R.G.) \\ 2 Department of Energy, Construction, Technology, Agriculture Division, Chamber of Agriculture Lower \\ Saxony, Mars-la-Tour-Str. 6, 26121 Oldenburg, Germany; harm.druecker@lwk-niedersachsen.de \\ 3 Department Plant Nutrition and Crop Production, Faculty of Agricultural Science and Landscape \\ Architecture, University of Applied Sciences, Am Krümpel 31, 49090 Osnabrück, Germany; \\ h-w.olfs@hs-osnabrueck.de \\ * Correspondence: mhorf@atb-potsdam.de; Tel.: +49-(0)-331-5699-441
}

Citation: Horf, M.; Vogel, S.; Drücker H.; Gebbers, R.; Olfs, H.-W. Optical Spectrometry to Determine Nutrient Concentrations and other

Physicochemical Parameters in Liquid Organic Manures: A Review. Agronomy 2022, 12, 514. https:// doi.org/10.3390/agronomy12020514

Academic Editor: Emad Jahanzad

Received: 23 January 2022

Accepted: 14 February 2022

Published: 18 February 2022

Publisher's Note: MDPI stays neutral with regard to jurisdictional claims in published maps and institutional affiliations.

Copyright: (c) 2022 by the authors. Licensee MDPI, Basel, Switzerland. This article is an open access article distributed under the terms and conditions of the Creative Commons Attribution (CC BY) license (https:// creativecommons.org/licenses/by/ $4.0 /)$

\begin{abstract}
Nutrient concentrations in livestock manures and biogas digestates show a huge variability due to disparities in animal husbandry systems concerning animal species, feed composition, etc. Therefore, a nutrient estimation based on recommendation tables is not reliable when the exact chemical composition is needed. The alternative, to analyse representative fertilizer samples in a standard laboratory, is too time- and cost-intensive to be an accepted routine method for farmers. However, precise knowledge about the actual nutrient concentrations in liquid organic fertilizers is a prerequisite to ensure optimal nutrient supply for growing crops and on the other hand to avoid environmental problems caused by overfertilization. Therefore, spectrometric methods receive increasing attention as fast and low-cost alternatives. This review summarizes the present state of research based on optical spectrometry used at laboratory and field scale for predicting several parameters of liquid organic manures. It emphasizes three categories: (1) physicochemical parameters, e.g., dry matter, $\mathrm{pH}$, and electrical conductivity; (2) main plant nutrients, i.e., total nitrogen, ammonium nitrogen, phosphorus, potassium, magnesium, calcium, and sulfur; and (3) micronutrients, i.e., manganese, iron, copper, and zinc. Furthermore, the commonly used sample preparation techniques, spectrometer types, measuring modes, and chemometric methods are presented. The primarily promising scientific results of the last 30 years contributed to the fact that near-infrared spectrometry (NIRS) was established in commercial laboratories as an alternative method to wet chemical standard methods Furthermore, companies developed technical setups using NIRS for on-line applications of liquid organic manures. Thus, NIRS seems to have evolved to a competitive measurement procedure, although parts of this technique still need to be improved to ensure sufficient accuracy, especially in quality management.
\end{abstract}

Keywords: chemometrics; farmyard manures; digestates; regression models; VIS-NIR spectra; MIR

\section{Introduction}

Animal manures and digestates from biogas production contain all essential plant nutrients crucial for growing crops to produce food, feed, and fibres [1], i.e., macronutrients such as nitrogen (usually given as total nitrogen (TN) and ammonium nitrogen $\left(\mathrm{NH}_{4}-\mathrm{N}\right)$ representing the directly plant-available fraction), phosphorus $(\mathrm{P})$, potassium $(\mathrm{K})$, magnesium (Mg), calcium (Ca), and sulfur (S) as well as micronutrients such as manganese $(\mathrm{Mn})$, iron $(\mathrm{Fe})$, copper $(\mathrm{Cu})$, and zinc $(\mathrm{Zn})$. In contrast to mineral fertilizers, farm-based liquid organic manures have several advantages. As residues from animal husbandry or biogas plants, they are virtually available for free on many farms or at least much cheaper 
compared to commercial fertilizers. Furthermore, the application of liquid organic manures increases the content of organic matter in the soil, thus leading to a better water storage capacity and overall improved soil fertility [2]. However, it is challenging to exactly quantify nutrient concentrations of these farm-based fertilizers due to inherent heterogeneity showing a solid and a liquid phase. Moreover, their composition depends on a wide variety of factors, e.g., animal species and age, feedstuff compositions, storage management, homogenization techniques, and stage of pumping. Presently, most farmers worldwide use simple recommendation tables for nutrient estimation, which have been compiled for several types of manures and digestates based on laboratory analyses during the last few decades. However, these tables do not cover the real variability of nutrient concentrations due to the diverse processes in animal husbandry. For this reason, the remaining uncertainty of real nutrient concentrations can tempt farmers to rather overdose organic fertilizers to ensure sufficient nutrient supply to the crop instead of risking productivity losses. In general, a nutrient surplus (especially of nitrate and phosphate) may lead to emissions of nutrients to non-agricultural ecosystems such as ground and surface waters, resulting in environmental problems such as eutrophication [3] as well as health problems, especially for infants, if nitrate reaches high amounts in drinking water.

As an alternative to following a general nutrient estimation, farmers can sample each livestock building or storage facility for liquid organic manures and send a more or less representative sample to a certified laboratory for accurate nutrient analysis. However, this procedure is rather time- and cost-intensive and needs a thorough homogenization before sampling and subsampling. Otherwise, measured nutrient concentrations from the laboratory may differ distinctly from true values [4].

Obviously, there is a high demand for reliable, rapid, and low-cost methods for quantifying nutrients in liquid organic manures. In contrast to recommendation tables and traditional wet chemistry-based analyses in certified laboratories, spectrometric methods might be a feasible alternative, if they attain a sufficient accuracy. Due to their practicability, farmers or service providers would be able to directly sample and analyse manures and digestates even on-farm or on-line during field application. Furthermore, in the case of on-farm analysis, farmers could analyse more samples from different parts and depths of the more or less homogenized storage tank, instead of sending only one sample for the total storage facility to a certified laboratory, resulting in a more representative nutrient quantification [5].

Due to the principal success of spectroscopic methods to predict nutrients in liquid organic fertilizers during the past three decades, these methods are established in some laboratories as alternative measurement techniques for liquid organic manures. Furthermore, during the last five years, on-line measurements of liquid manures at farm and field scale were successfully proven for dry matter and the main nutrients $\mathrm{N}$ (total $\mathrm{N}$ and ammonium N), P, and K [6]. These recent developments necessitate reviewing the benefit of optical spectrometry for characterizing liquid manures, based on an extensive literature survey. Therefore, the reader is introduced to the methodical know-how of spectrometric measurements of liquid organic manures. Afterward, spectrometric studies on liquid manures of over 40 publications are comparatively evaluated; the corresponding sample preparation techniques, spectrometer types, measuring modes, and chemometric methods are summarized; and the best performing methodical approaches are discussed.

\section{Spectrometric Methods}

\subsection{Physical Background}

Predicting physicochemical parameters and nutrient concentrations of liquid manures and digestates by ultraviolet (UV), visual (VIS), near-infrared (NIR), or mid-infrared (MIR) spectrometry requires element-specific interactions of their chemical components with electromagnetic radiation. Valence electrons are able to absorb and re-emit light in the UV (200-380 nm) and VIS (380-780 nm) regions. Radiation of the NIR region $(780-2500 \mathrm{~nm})$ interacts with overtone and combination vibrations of molecule bindings (e.g., $\mathrm{O}-\mathrm{H}, \mathrm{N}-\mathrm{H}$, and $\mathrm{C}-\mathrm{H}$ ) belonging to fundamental vibrations of molecular bindings in the MIR region $(2500-25,000 \mathrm{~nm})$ such as 
stretching (symmetric or antisymmetric), bending, wagging, rocking, or twisting $[7,8]$. Furthermore, when metal ions (e.g., $\mathrm{Fe}, \mathrm{Cu}, \mathrm{Zn}$ ) form coordinative bonds with organic compounds (so-called ligands), the conjugated $\pi$-electron system of the formed complex is able to absorb photons from the UV to the NIR region [9]. Another, though less investigated, possibility is that the characteristic of cations (e.g., $\mathrm{Mg}^{2+}, \mathrm{Ca}^{2+}$ ) to form hydrate envelopes can also cause band shifts by influencing the hydrogen bonding structure of the sample, which can be identified by comprehensive chemometric analysis [10].

A second reason why it is possible to predict chemical contents of liquid organic fertilizers with optical spectrometry is a sometimes strong correlation of spectrally inactive components (e.g., metals such as $\mathrm{Ca}, \mathrm{Mg}, \mathrm{Cu}, \mathrm{Zn}$ ) with at least one spectrally active compound [11].

In comparison to MIR spectrometry, NIR has the advantage that light of these wavelengths is able to penetrate deeper into the sample [12]. This might result in more representative measurements for liquid organic manures because of their naturally heterogeneous character. On the other hand, absorption bands resulting from overtone and combination vibrations in the NIR region are less intensive (by a factor of 10-100) than those of fundamental vibrations in the MIR region, leading to higher variations in detector signals which might result in a less precise quantification [10].

\subsection{Spectrometer Types}

Up to now, at least four main types of NIR spectrometers are utilized in published papers to analyse liquid organic manures using spectra of the near-infrared region:

- Dispersive grating scanning monochromator (SM) benchtop spectrometers, which are characterized by a high resolution but slow scan speed and sensitive moving parts, limiting the on-site use at the farm or in the field (e.g., NIR-Systems 6500, FOSS, Hilleroed, Denmark, used in [13-20]);

- $\quad$ Dispersive diode array (DA) spectrometers with a high scan speed and a robust apparatus either as benchtop or as portable version for lab and on-site applications (e.g., Corona 45, Carl Zeiss Spectroscopy GmbH, Jena, Germany, used in [11,21-24]);

- $\quad$ Fourier transform (FT) spectrometers, which have the advantage of being faster and higher in resolution compared to dispersive spectrometers. Originally they were used only for the mid-infrared region, but today they are also well established in the nearinfrared region (e.g., NIRFlex N-500, BÜCHI Labortechnik AG, Flawil, Switzerland, used in $[21,25,26])$;

- $\quad$ Bandpass spectrometers using bandpass filters for selected wavelengths, characterized by low-cost detectors with small wavelength ranges (e.g., MM55 instrument, NDC Infrared Engineering Ltd., Maldon, England, used in [12]).

Up to now, only two different MIR spectrometers were tested to analyse manure properties with spectra of the mid-infrared region. These Fourier transform MIR-instruments were either equipped with:

- The photoacoustic (PA) technique, which records the sound of mid-infrared radiation emitted by irradiated samples due to pressure and volume changes (Nicolet 380 FT-IR, Thermo Fisher Scientific Inc., Waltham, MA, USA, combined with the photoacoustic cell PAC300, MTEC Photoacoustics Inc., Ames, IA, USA, used in [27]);

- The attenuated total reflection (ATR) technique (MonitIR ATR spectrometer, SpectraTech Applied Systems Inc., Shelton, CT, USA, used in [12]), which measures the absorption of evanescent waves that penetrate only a few nanometres into the sample behind a totally reflecting prism surface [8].

\subsection{Measuring Modes and Sample Preparation Techniques}

The interaction of radiation with components of liquid manures leads to different fractions of light reflection, transmission, and absorption. The latter can only be determined indirectly by measuring the reflection and/or transmission. One challenge that spectrometric methods with liquid materials such as manures or digestates have to deal with is 
the high impact of light absorption by water above $1000 \mathrm{~nm}$. Thus, only a few percent of the incoming light is reflected in the near- and mid-infrared regions. Because of liquid manures' strongly absorbent character with very little light transmission, the measurement of reflection is the mode of choice. Measuring slurries in reflection mode has the advantage that the reflected light is not influenced by varying sample thickness.

It is also possible to measure in transflection mode (a combination of transmission and reflection), in which transmitted light is backscattered by a white standard or golden reflector and transmitted back through the sample to the detector. An appropriate sample thickness for this technical set-up is about $1 \mathrm{~mm}$, which means an optical pathway of $2 \mathrm{~mm}$ [18]. Finzi et al. [25], Malley et al. [28-30], Malley et al. [14], and Saeys et al. [18] published data for measuring animal manure in transflection mode.

The distinct heterogeneity of liquid organic manures is another challenge for spectrometric measurements. These fertilizers are a suspension of solid particles in a liquid matrix, separating during storage due to different densities [31]. According to Saeys [5], the solid particles sink downward (e.g., in pig and poultry manures) or float up to the top of the liquid phase (e.g., in cattle manures) depending on the type of organic fertilizer. This is a substantial disadvantage for carrying out representative measurements because both fractions have different characteristics. While the solid phase contains mainly organic matter with higher concentrations of phosphorus, calcium, magnesium, and organically bound nitrogen, the liquid phase contains most of the ammonium nitrogen and potassium [5]. Thus, it is very important for an accurate determination of nutrients to obtain a representative sample of the liquid organic fertilizers and to obtain a homogeneous sample during measurement. Usually, this problem is overcome by mixing the slurry sample right before measurement, e.g., by using a spatula [32], a disperser [21,33], or a magnetic stirrer [24] or by shaking the liquid manure several times in a plastic bag [19]. Another possibility to eliminate segregation processes during spectrometric measurements in the lab is to reduce the water content either by air-drying [22,27] or by using a drying cabinet [17,34]. Finzi et al. [25] separated the solid and liquid phases by filtering the sample with a $1 \mathrm{~mm}$ mesh followed by analysing the eluate. A rather new alternative approach is the utilization of so-called Nanobags, which are filled with a strongly absorbing carrier material based on zeolite. After adding liquid organic manures into the bags, the carrier material with the adhering organic fertilizers is dried and ground to obtain a homogenized material for spectroscopic analysis ([35,36]; see also Section 4).

\subsection{Spectra of Liquid Organic Manures}

The reflected light of a sample has to be set into relation to a reference reflection standard with known reflection properties. This ratio is called reflectance $(R)$ and is displayed in \% (Figure 1). However, about one-quarter of the studied literature prefers a conversion of reflectance into a pseudo absorbance (pA; Equation (1)) analogue to the absorbance (A; Equation (2); also called extinction or optical density) of the Lambert-Beer law from 1852, where absorbance is directly proportional to the concentration of dissolved components with absorbing properties. The difference between absorbance and pseudo absorbance is that $\mathrm{pA}$ is calculated by the measured diffuse reflectance instead of transmittance $(\mathrm{T})$. Up to now, the term "pseudo absorbance" is rarely used in literature (e.g., in [37]). Unfortunately, it is often mistaken with the term "absorbance". Furthermore, there is no mathematical evidence that the transformation of reflectance spectra into pseudo absorbance spectra has any advantages. The assumption that diffuse reflectance is also directly proportional to the concentration of dissolved components, as is the case for absorbance, is wrong because diffuse reflectance is not directly correlated to transmittance but also depends on the absorptance $(\alpha$; Equation (3)) and scattering properties of the surface.

$$
\begin{aligned}
\mathrm{pA} & =\log (1 / \mathrm{R}) \\
\mathrm{A} & =\log (1 / \mathrm{T})
\end{aligned}
$$




$$
\mathrm{R}=1-\mathrm{T}-\alpha
$$

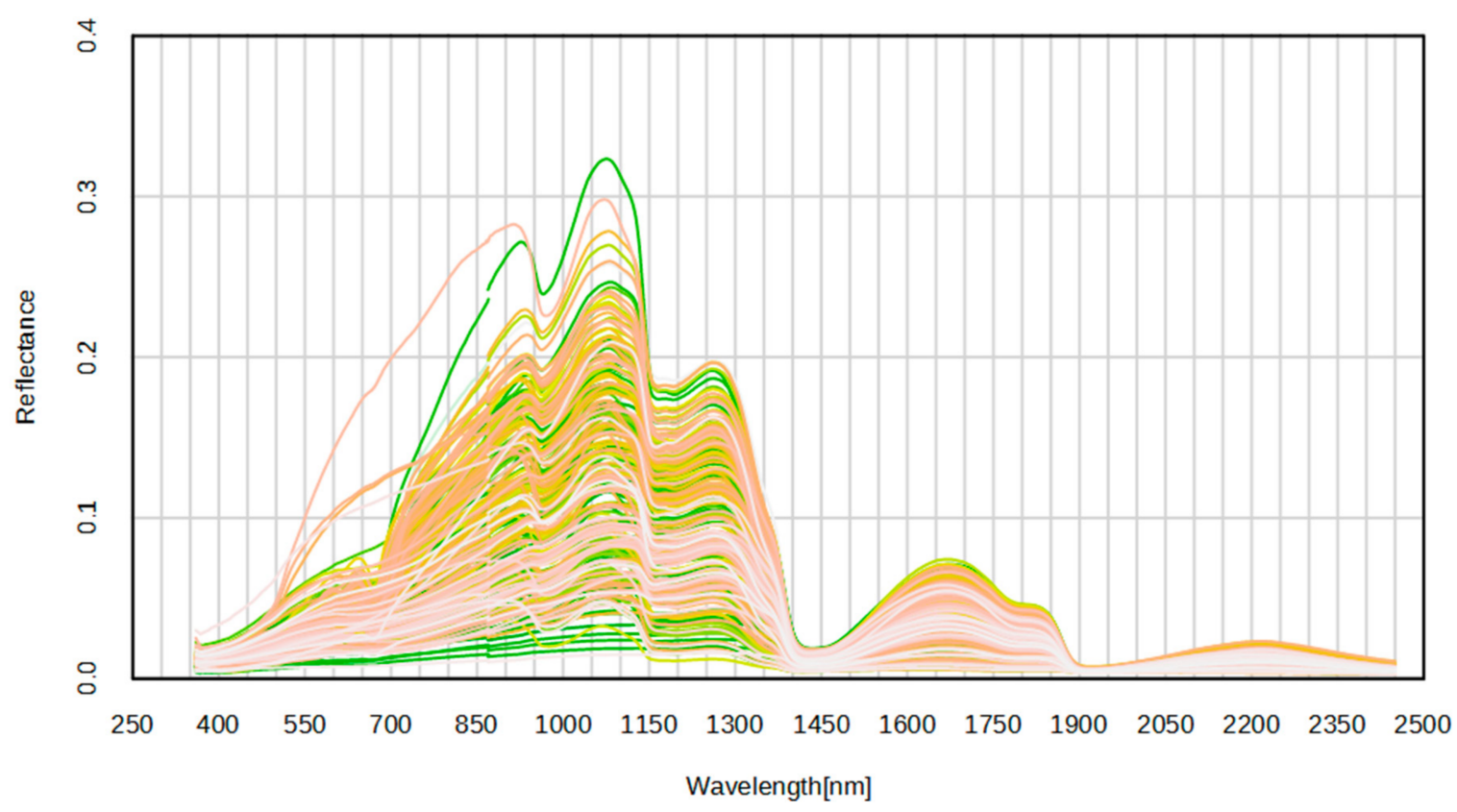

Figure 1. Typical VIS-NIR reflectance spectra for liquid organic manures (pig, cattle, digestate) from a study conducted in north Germany (detected with UV-VIS-NIR fibered ArcOptix spectrometer, ArcOptix, Neuchatel, Switzerland).

Because of their dark brown colour, liquid animal manures and biogas digestates absorb light in the whole region of visible wavelengths $(380-780 \mathrm{~nm})$, decreasing to longer wavelengths in the red region up to $780 \mathrm{~nm}$ and resulting in increasing reflectances (Figure 1). The strong valleys around $675 \mathrm{~nm}$ in many samples probably correspond to chlorophyll, which is typical for dairy or cattle manure and rather unusual for hog manure, because forages are rarely used in pig nutrition [24]. The highly pronounced valleys around 1440 and $1920 \mathrm{~nm}$ are the result of a strong absorption of radiation by water molecules, which belong to both the first overtone vibration of the fundamental stretching vibration and the combination vibration of the fundamental stretching and bending vibration of the two O-H molecule bonds [38].

\subsection{Statistical Analysis Using Chemometric Methods}

\subsubsection{Preprocessing Methods}

Several different preprocessing methods were tested on the whole spectra or selected wavelengths of the spectra of liquid organic fertilizers to correct signals for noise, to remove baseline shifts, or to reduce data size before conducting a statistical analysis, including the following:

- $\quad$ Mean centering (MC; Equation (4));

- Smoothing, e.g., rectangular smooth (Sr; unweighted sliding average smooth with $\mathrm{n}$ points; Equation (5)) or Savitzky-Golay smoothing (SG0; [39]);

- Standard normal variate transformation/scaling (SNV; Equation (6); [40]);

- Standard normal variate transformation combined with detrend (SNVD; used in [19]);

- First or second derivate, e.g., with Savitzky-Golay algorithm (SG1 or SG2; [39]);

- Multiplicative signal/scatter correction (MSC; [41]; Equation (7));

- Extended multiplicative scattering correction (EMSC; developed by [42]; used in [21]);

- A variant of orthogonal signal correction (OSC; [43]), called direct orthogonal signal correction (DOSC; developed by [44]; used in [45]);

- Area normalization (used in [12]), where each sample is normalized to the area under the spectra (calculated as integral of the spectral curve). 


$$
\begin{gathered}
M C=\left(y_{w}-\bar{y}\right) \\
S_{r \text { with } n-\text { points }}=\frac{y_{w-\frac{n}{2}+1}+\ldots+y_{w-1}+y_{w}+y_{w+1}+\ldots+y_{w+\frac{n}{2}-1}}{n} \\
S N V_{w}=\frac{M C}{\sigma}=\left(y_{w}-\bar{y}\right) / \sqrt{\frac{\sum\left(y_{w}-\bar{y}\right)^{2}}{N-1}} \\
M S C=\frac{y_{w}-a_{i}}{b_{i}}
\end{gathered}
$$

where:

$y_{w}=$ absorbance at a specific wavelength;

$\bar{y}=$ mean value of all measured values of a spectrum;

$a_{i}=$ additive effect calculated by ordinary least square regression (OLS) for each spectrum on the mean spectrum of all spectra;

$b_{i}=$ multiplicative effect calculated by ordinary least square regression (OLS) for each spectrum on the mean spectrum of all spectra.

The preprocessing methods are used to eliminate spectral noise or other disturbing effects such as baseline shift; however, it has to be kept in mind that the effectiveness of a preprocessing method depends on manure type and sample set.

\subsubsection{Regression Models and Machine Learning Methods}

After the preprocessing of the spectral data, a regression model is built. The aim is to find a mathematical correlation between the spectra (predictor or independent variables) and the corresponding reference values (response or dependent variables) to subsequently predict the values of all relevant components in liquid organic manures solely based on a corresponding spectrum. For that, the method of partial least square regression (PLSR) introduced by Wold in 1975 [46] is frequently utilized to predict nutrients in liquid organic manures with optical spectrometry. In PLSR, the predictor variables (i.e., the wavelengths of VIS-NIR spectra) are reduced to a few uncorrelated components, similar to a principal component analysis (PCA). Afterward, least square errors are determined for calculating the best regression equation with each response variable. This method is often applied when having many highly collinear predictor variables in conjunction with a small number of samples, as is usually the case in VIS-NIR spectrometry [47].

Another applied prediction model is called multiple linear regression (MLR), which is in principle the extension of ordinary least square regression (OLSR) by including more than one predictor variable. This concept was first introduced by Sternberg et al. in 1960 [48]. Dagnew et al. [49] and Malley et al. [14,28-30,50] applied this method to predict nutrient concentrations in liquid manures, but it is rarely used by any other author in this context. Chen et al. [51] compared a machine learning method called artificial neuronal networks (ANNs) with PLSR on spectra of poultry manures and found an overall improvement for $\mathrm{TN}, \mathrm{NH}_{4}-\mathrm{N}, \mathrm{P}$, and $\mathrm{K}$ using ANNs.

Further regression techniques utilized for processing spectral data are least absolute shrinkage and selection operator (LASSO; [52]) regression, ridge regression (RR; [53]), least angle regression (LAR; [54]), random forest (RF; [55]), and forward stagewise or stepwise regression. However, none of these algorithms were as yet used in published data for livestock manures and digestates.

\subsubsection{Model Evaluation Parameters}

To evaluate the quality of prediction models of NIR data for specific dependent variables, several different quality factors are used, such as:

- Coefficient of correlation (R);

- Coefficient of determination $\left(\mathrm{R}^{2}\right.$; Equation (8));

- $\quad$ Standard error of prediction, calibration, or cross-validation (SEP, SEC, SECV; Equation (9)); 
- Root mean square error of prediction or cross-validation (RMSEP/RMSECV; also called root mean squared deviation (RMSD); [56,57]). RMSEP is almost the same as SEP, which is additionally bias-corrected (Equation (10));

- Ratio of the standard deviation of the reference values ( $\mathrm{SD}_{\text {ref }}$; Equation (11)) and the SEP (SD ref /SEP; so-called ratio of performance (RPD) introduced by [58]);

- Ratio of the sample range and RMSEP (so-called range error ratio (RER), e.g., used in [24]);

- Ratio of relative mean squared difference (RMSD) and mean (so-called relative standard deviation (RSD), e.g., used in [16]);

- Ratio of sample range and SEP (e.g., [15]);

- The slope (so-called residual variance or random error) and the bias (so-called error of means or systematic error) of the regression line.

In many publications, both $\mathrm{R}^{2}$ and RDP were chosen as quality indicators. However, Minasny and McBratney [59] explained the redundancy of publishing both parameters because of their mathematic relationship (Equation (12)) and hence identical significance. Nevertheless, all of these evaluation indicators assume a normal distribution of the measured sample concentrations. However, there is a frequent occurrence of log-normal distributed sample concentrations, which was the reason Bellon-Maurel et al. [60] proposed a new index called the ratio of performance to interquartile distance (RPIQ; Equation (13)). This ratio is based on the first $\left(Q_{1}\right)$ and third $\left(Q_{3}\right)$ quartiles of the empirical distribution and thus considers the increased appearance of samples with smaller concentrations in a log-normal distributed sample set.

$$
\begin{gathered}
R^{2}=\frac{\sum_{i}^{N}\left(\hat{y}_{i}-\bar{y}\right)^{2}}{\sum_{i}^{N}\left(y_{i}-\bar{y}\right)^{2}} \\
S E P=\sqrt{\sum_{i=1}^{N} \frac{\left(\hat{y}_{i}-y_{i}-\text { Bias }\right)^{2}}{N-1}} ; \text { Bias }=\bar{y}-\overline{\hat{y}} \\
R M S E P=\sqrt{\sum_{i=1}^{N} \frac{\left(\hat{y}_{i}-y_{i}\right)^{2}}{N}} \\
S D_{r e f}=\sqrt{\sum_{i=1}^{N} \frac{\left(y_{i}-\bar{y}\right)^{2}}{N-1}} \\
R D P=\frac{S D_{r e f}}{S E P}=\frac{1}{\sqrt{1-R^{2}}} \\
R P I Q=\frac{Q_{3}-Q_{1}}{S E P}
\end{gathered}
$$

where:

$N$ = number of samples;

$y i=$ ith reference value;

$\hat{y} i=$ ith predicted value;

$\bar{y}=$ mean of all reference values;

$\overline{\hat{y}}=$ mean of all predicted values.

\subsubsection{Outliers}

Several methods have been proposed to detect outliers in the data, although no commonly accepted definition exists for what constitutes an outlier. In general, outliers can be distinguished in "reference outliers" (conspicuous reference values determined by standard laboratory methods), "spectral outliers" (conspicuous spectra), and "prediction outliers" (conspicuously deviating values calculated by chemometric methods). In the evaluated literature, several different methods were used to detect these outliers. 
Althaus et al. [13] defined all sample spectra with a so-called "global $H^{\prime \prime}$ value $(\mathrm{GH})>3.2$ and a so-called "neighbourhood $\mathrm{H}^{\prime \prime}$ value $(\mathrm{NH})>1.2$ as spectral outliers. $\mathrm{GH}$ and NH are special parameters, calculated by the chemometric software WinISI (FOSS), whereas GH is very similar to the standardized Mahalanobis distance $\mathrm{H}$ (distance of a sample point to the mean) and $\mathrm{NH}$ is a kind of relation to neighbouring sample points. Sørensen et al. [19] defined all sample spectra with a GH value $>3.5$ as spectral outliers. For prediction outliers, he used a T value (Student's $t$-test) $>3$ of predicted concentrations, which means that the residual of the predicted sample concentration to the regression line is more than 3 times the RMSECV or RMSEP of the sample set (Studentized residual). Similar to the $t$-test, Reeves [17] calculated the absolute difference of predicted and reference values of the samples and defined all values as predicted outliers that were higher than 3 times the standard deviation (SD) from the mean difference. Cabassi et al. [21] and De Ferrari et al. [33] detected outliers by principal component analysis on PLS principal components using Dixon's Q-test and Hoteling's $\mathrm{T}^{2}$ test with a 95\% confidence limit, which is about 2 times the SD. Another possible, but more subjective, method is plotting Cook's distances vs. leverage values [34] or plotting PCA scores vs. leverage values [15] and deleting conspicuous deviations.

\section{Published Data}

Tables 1-5 give a detailed overview summarizing published correlation coefficients $\left(\mathrm{R}^{2}\right)$ and standard errors of predictions (RMSEP or SEP) for nutrient concentrations and physicochemical parameters of liquid organic manures, determined via spectrometric and standard laboratory procedures. In comparison to liquid livestock manures, the number of published papers dealing with spectrometrically measured data for biogas digestates is very small. In Table 6, the data set is further condensed and the published ranges of $R^{2} s$ and RMSEPs for each parameter are indicated. 
Table 1. Summary of published data for predicting parameters in liquid organic manures based on optical spectroscopy. Additionally, publications about solid organic manures such as solid poultry manure or dairy faeces are included.

\begin{tabular}{|c|c|c|c|c|c|c|c|c|c|c|c|}
\hline Reference & $\begin{array}{c}\text { Althaus et al. } \\
2013 \text { [13] }\end{array}$ & $\begin{array}{l}\text { Asai et al. } \\
1993 \text { [61] }\end{array}$ & $\begin{array}{c}\text { Becaccia et al. } \\
2015[62]\end{array}$ & $\begin{array}{c}\text { Benozzo et al. } \\
2018 \text { [63] }\end{array}$ & $\begin{array}{c}\text { Cabassi et al. } \\
2015 \text { [21] }\end{array}$ & $\begin{array}{l}\text { Chen et al. } \\
2009 \text { [51] }\end{array}$ & $\begin{array}{l}\text { Chen et al. } \\
2010 \text { [45] }\end{array}$ & $\begin{array}{c}\text { Dagnew et al. } \\
2004[49]\end{array}$ & $\begin{array}{c}\text { De Ferrari } \\
\text { et al. } 2007[33]\end{array}$ & $\begin{array}{l}\text { Dolud et al. } \\
2005 \text { [64] }\end{array}$ & $\begin{array}{l}\text { Dong et al. } \\
2011 \text { [34] }\end{array}$ \\
\hline $\begin{array}{l}\text { Liquid } \\
\text { organic } \\
\text { manure }\end{array}$ & $\begin{array}{l}\text { dried dairy } \\
\text { faeces }\end{array}$ & $\begin{array}{l}\text { dried dairy } \\
\text { cattle }\end{array}$ & pig & digestate & cattle & poultry & poultry & hog & cattle & hog & dried poultry \\
\hline $\begin{array}{l}\text { No. samples } \\
\text { (\% for } \\
\text { validation) }\end{array}$ & $168(14 \%)$ & $75(33 \%)$ & $79(-)$ & $80(-)$ & $99(33 \%)$ & $91(25 \%)$ & $91(25 \%)$ & $88(28 \%)$ & $101(-)$ & $128(40 \%)$ & $74(66 \%)$ \\
\hline $\begin{array}{l}\text { Scan mode } \\
\text { (material) }\end{array}$ & $\mathrm{R}$ & $\mathrm{R}$ & R (PE-bags) & $\begin{array}{c}\mathrm{R} \\
\text { (boro-silicate) }\end{array}$ & $\mathrm{R}$ & $\mathrm{R}$ & $\mathrm{R}$ & $\mathrm{R}$ & $\mathrm{R}$ & $\mathrm{T}(3 \mathrm{~mm})$ & $\mathrm{R}$ \\
\hline Range (nm) & $408-2493$ & $1445-2348$ & $1100-2400$ & $950-1650$ & $\begin{array}{l}\text { (a) } 1000-2500 \\
\text { (b) } 1100-1690\end{array}$ & $1000-2500$ & $1000-2500$ & $250-2500$ & $1100-2498$ & $960-1690$ & $800-2500$ \\
\hline \multicolumn{12}{|c|}{$R^{2}$ (RMSEP or $\left.\underline{S E P}\right)$ of } \\
\hline DM (\%) & - & - & $0.96(0.18)$ & $0.66(0.63)$ & (a) 0.92 (1.01) & - & - & $0.92(0.80)$ & $0.95(0.95)$ & $0.97(0.28)$ & - \\
\hline $\mathrm{pH}$ & - & - & $0.84(\overline{0.11})$ & $0.64(0.09)$ & (2) & - & - & - & $0.29(0.25)$ & - & - \\
\hline $\mathrm{EC}(\mathrm{mS} / \mathrm{cm})$ & - & - & - & $0.86(1.41)$ & - & - & - & - & $0.11(2.55)$ & - & - \\
\hline $\mathrm{Na}(\mathrm{g} / \mathrm{kg})$ & - & - & - & - & - & - & - & - & - & - & - \\
\hline $\begin{array}{l}\text { OM/TC/TOC/ } \\
\text { ash (g/kg) }\end{array}$ & TC $0.96(\underline{2.90})$ & $\begin{array}{l}\text { ash } 0.99(22.4) \\
\text { TC } 0.97(14.5)\end{array}$ & ash $0.81(\underline{0.10})$ & TOC $0.73(2.1)$ & $\begin{array}{l}\text { (a) ash } 0.88 \\
(3.3)\end{array}$ & - & - & - & TC 0.92 (3.74) & - & - \\
\hline $\mathrm{NH}_{4}-\mathrm{N}(\mathrm{g} / \mathrm{kg})$ & $0.91(\overline{0.18})$ & - & $0.88(\underline{0.80})$ & $0.85(0.23)$ & (b) $0.78(0.27)$ & $0.90(0.75)$ & $0.88(0.85)$ & - & $0.83(0.18)$ & $0.88(\underline{0.24})$ & - \\
\hline$P(g / k g)$ & - & - & - & $0.80(0.12)$ & (b) $0.74(0.15)$ & $0.86(0.42)$ & $0.74(0.64)$ & $0.79(0.42)$ & $0.78(0.13)$ & - & $0.74(3.4) * 1$ \\
\hline $\mathrm{K}(\mathrm{g} / \mathrm{kg})$ & - & - & - & $0.73(0.12)$ & - & $0.72(0.73)$ & $0.57(1.08)$ & $0.68(0.50)$ & $0.19(0.67)$ & - & - \\
\hline$M g(g / k g)$ & - & - & - & - & - & - & - & - & - & - & - \\
\hline $\mathrm{Ca}(\mathrm{g} / \mathrm{kg})$ & - & - & - & $0.69(4.3)$ & - & - & - & - & - & - & - \\
\hline $\mathrm{S}(\mathrm{g} / \mathrm{kg})$ & - & - & - & $0.78(0.4)$ & - & - & - & - & - & - & - \\
\hline Mn (g/kg) & - & - & - & - & - & - & - & - & - & - & - \\
\hline $\mathrm{Fe}(\mathrm{g} / \mathrm{kg})$ & - & - & - & $0.81(0.6)$ & - & - & - & - & - & - & - \\
\hline $\mathrm{Cu}(\mathrm{g} / \mathrm{kg})$ & - & - & - & - & - & - & - & - & - & - & $0.90(0.01) * 1$ \\
\hline $\mathrm{Zn}(\mathrm{g} / \mathrm{kg})$ & - & - & - & $0.85(0.05)$ & - & - & - & - & - & - & $0.87(0.03) * 1$ \\
\hline
\end{tabular}


Table 2. Summary of published data for predicting parameters in liquid organic manures based on optical spectroscopy. Additionally, publications about solid organic manures such as solid poultry manure or dairy faeces are included.

\begin{tabular}{|c|c|c|c|c|c|c|c|c|c|c|c|}
\hline Reference & $\begin{array}{l}\text { Du et al. } \\
2010 \text { [27] }\end{array}$ & $\begin{array}{l}\text { Finzi et al. } \\
2015 \text { [25] }\end{array}$ & $\begin{array}{c}\text { Finzi et al. } \\
2015 \text { [25] }\end{array}$ & $\begin{array}{l}\text { Finzi et al. } \\
2015 \text { [25] }\end{array}$ & $\begin{array}{c}\text { Kemsley } \\
\text { et al. 2001 } \\
\text { [12] }\end{array}$ & $\begin{array}{c}\text { Kemsley } \\
\text { et al. 2001 } \\
{[12]}\end{array}$ & $\begin{array}{l}\text { Malley et al. } \\
\text { 2001a [28] }\end{array}$ & $\begin{array}{l}\text { Malley et al. } \\
\text { 2001b [29] }\end{array}$ & $\begin{array}{l}\text { Malley et al. } \\
\text { 2001c [30] }\end{array}$ & $\begin{array}{l}\text { Malley et al. } \\
2002 \text { [14] }\end{array}$ & $\begin{array}{l}\text { Malley et al. } \\
2005[22]\end{array}$ \\
\hline $\begin{array}{l}\text { Liquid organic } \\
\text { manure }\end{array}$ & $\begin{array}{l}\text { dried: pig, } \\
\text { poultry, } \\
\text { cattle }\end{array}$ & pig & dairy & digestate & $\begin{array}{l}\text { pig, turkey, } \\
\text { cattle }\end{array}$ & turkey, cattle & hog & hog & hog & hog & air-dried cattle \\
\hline $\begin{array}{l}\text { No. samples (\% } \\
\text { for validation) }\end{array}$ & $81(27 \%)$ & $12(-)$ & $12(-)$ & $12(-)$ & $45(-)$ & $30(-)$ & $80(-)$ & $80(-)$ & $80(-)$ & 64 or $75(50 \%)$ & $179(-)$ \\
\hline Scan mode & $\mathrm{PA}$ & $\mathrm{T}(0.3 \mathrm{~mm})$ & $\mathrm{T}(0.3 \mathrm{~mm})$ & $\mathrm{T}(0.3 \mathrm{~mm})$ & ATR & $\mathrm{R}$ & $\mathrm{T}(2 \mathrm{~mm})$ & $\mathrm{T}(2 \mathrm{~mm})$ & $\mathrm{T}(2 \mathrm{~mm})$ & $\mathrm{T}(2 \mathrm{~mm})$ & $\mathrm{R}$ \\
\hline \multicolumn{12}{|c|}{$R^{2}$ (RMSEP or $\left.\underline{S E P}\right)$ of } \\
\hline DM (\%) & - & $0.96(0.87)$ & $0.92(0.25)$ & $0.92(0.22)$ & - & - & $0.97 * 2(\underline{0.49})$ & $0.96(\underline{0.57})$ & - & $0.94(\underline{0.20}) * 3$ & - \\
\hline $\mathrm{pH}$ & - & - & - & - & - & - & $0.96(\underline{0.07)}$ & - & - & $0.84(0.09)$ & $0.89(\underline{0.29})$ \\
\hline $\mathrm{EC}(\mathrm{mS} / \mathrm{cm})$ & - & - & - & - & - & - & $0.94(1.17)$ & - & - & $0.85(\underline{\mathbf{0 . 7 7}})$ & - \\
\hline $\mathrm{Na}(\mathrm{g} / \mathrm{kg})$ & - & - & - & - & - & - & $0.85 * 2(0.03)$ & $0.61(\underline{0.05})$ & $0.80(0.04)$ & $0.95(\underline{0.03})$ & - \\
\hline $\begin{array}{l}\text { OM/TC/susp C } \\
(\mathrm{g} / \mathrm{kg})\end{array}$ & $0.93(23.8)$ & - & - & - & - & - & $\begin{array}{l}\text { susp } C 0.98 \\
(1.56)\end{array}$ & - & - & $\operatorname{susp} C 0.99(0.7)$ & TC $0.91(\underline{23.4})$ \\
\hline $\mathrm{NH}_{4}-\mathrm{N}(\mathrm{g} / \mathrm{kg})$ & - & $0.95(0.21)$ & $0.91(0.08)$ & $0.98(0.12)$ & $0.81(3.87)$ & $0.92(3.50)$ & $0.95(0.24)$ & - & - & $0.97(0.07)$ & - \\
\hline P/TDP (g/kg) & - & - & - & - & - & - & $0.98 * 2(0.21)$ & $0.85(0.57)$ & $0.94(0.35)$ & TDP $\mathbf{0 . 9 9 ( 0 . 0 6 )}$ & $0.61(0.70)$ \\
\hline$K(g / k g)$ & - & - & - & - & - & - & $0.92^{* 2}(\underline{0.16})$ & $0.62(\underline{0.32})$ & $0.75(0.24)$ & $0.87(\underline{0.06})$ & $0.83(\underline{2.9})$ \\
\hline $\mathrm{Mg}(\mathrm{g} / \mathrm{kg})$ & - & - & - & - & - & - & $0.98 * 2(0.10)$ & $0.86(0.27)$ & $0.96(0.14)$ & $0.98(0.03)$ & - \\
\hline $\mathrm{Ca}(\mathrm{g} / \mathrm{kg})$ & - & - & - & - & - & - & $0.97 * 2(\overline{0.21})$ & $0.74(0.63)$ & $0.90(0.35)$ & $0.80(\underline{\mathbf{0 . 0 3}})$ & - \\
\hline $\mathrm{S}(\mathrm{g} / \mathrm{kg})$ & - & - & - & - & - & - & $0.95 * 2(\underline{0.01})$ & $0.84(\underline{0.02})$ & $0.84(0.02)$ & - & $0.73(\underline{0.44})$ \\
\hline $\mathrm{Mn}(\mathrm{g} / \mathrm{kg})$ & - & - & - & - & - & - & $0.96 * 2(0.01)$ & $0.78(\underline{\mathbf{0 . 0 1}})$ & $0.88(\mathbf{0 . 0 1})$ & - & - \\
\hline $\mathrm{Fe}(\mathrm{g} / \mathrm{kg})$ & - & - & - & - & - & - & $0.96 * 2(0.03)$ & $0.77(\overline{0.06})$ & $0.97(0.02)$ & - & - \\
\hline $\mathrm{Cu}(\mathrm{g} / \mathrm{kg})$ & - & - & - & - & - & - & $0.99 * 2(\overline{0.01})$ & $0.92(\underline{\mathbf{0 . 0 1}})$ & $0.95(\mathbf{0 . 0 1})$ & - & - \\
\hline $\mathrm{Zn}(\mathrm{g} / \mathrm{kg})$ & - & - & - & - & - & - & $0.98 * 2(0.02)$ & $0.79(\overline{0.06})$ & $0.93(\mathbf{0 . 0 0 1})$ & - & - \\
\hline
\end{tabular}

Best values for each parameter are highlighted in bold letters, dried samples in red, digestate samples in green, MIR technique in blue, and SEP values in underlined letters. Scan mode: $\mathrm{R}=$ reflectance, $\mathrm{T}=$ transflectance, $\mathrm{ATR}=$ attenuated total reflection, $\mathrm{PA}=$ photoacoustic; Parameters: $\mathrm{DM}=$ dry matter, $\mathrm{EC}=$ electrical conductivity, $\mathrm{OM}=$ organic matter, TC $=$ total carbon susp $\mathrm{C}=$ suspended carbon, $\mathrm{TN}=$ total nitrogen, $\mathrm{TDN}=$ total dissolved nitrogen, $\mathrm{TP}=$ total phosphorus, $\mathrm{TDP}=$ total dissolved phosphorus; $\mathrm{R}^{2}=$ coefficient of determination, $\mathrm{RMSEP}=$ root mean squared error of prediction, $\mathrm{SEP}=$ standard error of prediction. ${ }^{* 2}$ Not published in Malley et al. (2001a) [28], but later in Malley et al. (2001c) [30]. ${ }^{* 3}$ DM calculated from parameter moisture $(\mathrm{m})$ by $\mathrm{DM}=1-\mathrm{m}$. 
Table 3. Summary of published data for predicting parameters in liquid organic manures based on optical spectroscopy. Additionally, publications about solid organic manures such as solid poultry manure or dairy faeces are included.

\begin{tabular}{|c|c|c|c|c|c|c|c|c|c|c|c|}
\hline Reference & $\begin{array}{l}\text { Millmier et al. } \\
2000[15]\end{array}$ & $\begin{array}{l}\text { Millmier et al. } \\
2000 \text { [15] }\end{array}$ & $\begin{array}{l}\text { Millmier et al. } \\
2000 \text { [15] }\end{array}$ & $\begin{array}{l}\text { Mouazen et al. } \\
2005 \text { [23] }\end{array}$ & $\begin{array}{c}\text { Reeves \& Van } \\
\text { Kessel, 2000a } \\
\text { [56] }\end{array}$ & $\begin{array}{c}\text { Reeves \& Van } \\
\text { Kessel, 2000b } \\
\text { [65] }\end{array}$ & $\begin{array}{c}\text { Reeves 2001a } \\
{[16]}\end{array}$ & $\begin{array}{c}\text { Reeves 2001a } \\
{[16]}\end{array}$ & $\begin{array}{c}\text { Reeves 2001b } \\
\text { [17] }\end{array}$ & $\begin{array}{l}\text { Saeys et al. } \\
2004 \text { [24] }\end{array}$ & $\begin{array}{c}\text { Saeys et al } \\
\text { 2005a [11] }\end{array}$ \\
\hline $\begin{array}{l}\text { Liquid } \\
\text { organic } \\
\text { manure }\end{array}$ & pig & $\begin{array}{l}\text { pig, lagoon } \\
\text { effluent }\end{array}$ & cattle & hog & dairy & dairy & dried poultry & dried poultry & poultry & hog & pig \\
\hline $\begin{array}{l}\text { No. samples } \\
\text { (\% for } \\
\text { validation) }\end{array}$ & $174(-)$ & $100(-)$ & $100(-)$ & $195(-)$ & $107(-)$ & $107(-)$ & $124(-)$ & $124(-)$ & $207(-)$ & $169(-)$ & $584(28 \%)$ \\
\hline $\begin{array}{l}\text { Scan mode } \\
\text { (material) }\end{array}$ & R (PE-bag) & R (PE-bag) & R (PE-bag) & $\mathrm{R}$ & R (PE-bag) & $\begin{array}{l}\mathrm{R} \text { (optic fibre } \\
\text { glass) }\end{array}$ & $\mathrm{R}$ & $\mathrm{R}$ & R (PE-bag) & $\begin{array}{l}\mathrm{R} \text { (quartz } \\
\text { glass) }\end{array}$ & $\begin{array}{l}\mathrm{R} \text { (quartz } \\
\text { glass) }\end{array}$ \\
\hline Range (nm) & $400-2498$ & $400-2498$ & $400-2498$ & $300-1700$ & $400-2498$ & $400-2300$ & $400-2498$ & $\begin{array}{l}\text { 2500-25,000 } \\
\quad \text { (MIR) }\end{array}$ & $\begin{array}{l}400-2498 \text { or } \\
1100-2498\end{array}$ & $400-1700$ & $306-1710$ \\
\hline \multicolumn{12}{|c|}{$R^{2}$ (RMSEP or SEP) of } \\
\hline DM (\%) & $0.85(\underline{1.58})$ & $0.90(\underline{0.08})$ & $0.91(\underline{3.61})$ & $0.91(1.24)$ & $0.95(1.02)$ & $0.96(0.91)$ & - & - & $0.85(11.3) * 5$ & $0.58(2.52)$ & $0.91(1.20)$ \\
\hline $\mathrm{pH}$ & - & - & - & - & - & - & - & - & - & $0.40(0.18)$ & - \\
\hline $\mathrm{Na}(\mathrm{g} / \mathrm{kg})$ & - & - & - & $0.52(0.49)$ & - & - & - & - & - & $0.40(0.29)$ & - \\
\hline OM/TC (g/kg) & - & - & - & $0.89(9.73)$ & TC $0.95(4.00)$ & TC $0.94(4.50)$ & - & - & - & $0.57(18.25)$ & $0.90(9.29)$ \\
\hline TN (g/kg) & $0.81(\underline{27.7})^{* 4}$ & $0.69(33.6) * 4$ & $0.67(\underline{2.1})^{* 4}$ & $0.89(1.22)$ & $0.96(0.30)$ & $0.94(0.33)$ & - & - & $0.89(3.43) * 4$ & $0.75(1.53)$ & $0.86(1.23)$ \\
\hline$P(g / k g)$ & $0.47(\underline{5.1}) * 4$ & $0.61(\underline{4.4})^{* 4}$ & $0.58(\underline{1.3}) * 4$ & $0.85(0.90)$ & - & - & $0.69(5.31) * 4$ & $0.82(3.98) * 4$ & $0.62(4.77) * 4$ & $0.59(0.54)$ & $0.75(1.12)$ \\
\hline $\mathrm{K}(\mathrm{g} / \mathrm{kg})$ & $0.79(\overline{20.3}) * 4$ & $0.71(22.3) * 4$ & $0.82(\overline{1.7}) * 4$ & $0.84(0.98)$ & - & - & $0.66(3.21)^{* 4}$ & $0.59(3.55) * 4$ & $0.63(3.23) * 4$ & $0.73(0.81)$ & $0.69(1.15)$ \\
\hline $\operatorname{Mg}(\mathrm{g} / \mathrm{kg})$ & - & - & - & $0.87(0.41)$ & - & - & $0.72(1.31)^{* 4}$ & $0.69(1.40) * 4$ & $0.46(1.36)^{* 4}$ & $0.61(0.35)$ & $0.80(0.50)$ \\
\hline Ca (g/kg) & - & - & - & $0.76(1.37)$ & - & - & $0.96(6.48)^{* 4}$ & $0.97(6.42) * 4$ & $0.80(8.27)^{* 4}$ & $0.52(0.86)$ & 0.59 (1.49) \\
\hline$S(g / k g)$ & - & - & - & - & - & - & $0.36(2.58)^{* 4}$ & $0.54(2.27) * 4$ & $0.59(1.87) * 4$ & - & $0.36(0.26)$ \\
\hline $\operatorname{Mn}(\mathrm{g} / \mathrm{kg})$ & - & - & - & - & - & - & $0.59(0.07) * 4$ & $0.63(0.06) * 4$ & $0.57(0.06)^{* 4}$ & - & - \\
\hline $\mathrm{Fe}(\mathrm{g} / \mathrm{kg})$ & - & - & - & - & - & - & - & - & - & - & - \\
\hline $\mathrm{Cu}(\mathrm{g} / \mathrm{kg})$ & & - & - & - & - & - & $0.57(0.21)^{* 4}$ & $0.66(0.13) * 4$ & $0.52(0.12) * 4$ & - & - \\
\hline Zn (g/kg) & - & - & - & - & - & - & $0.56(0.10) * 4$ & $0.12(0.15) * 4$ & $0.50(0.09) * 4$ & - & - \\
\hline
\end{tabular}

Best values for each parameter are highlighted in bold letters, dried samples in red, MIR technique in blue, and SEP values in underlined letters. Scan mode: $\mathrm{R}=$ reflectance; Parameters $\mathrm{DM}=$ dry matter, $\mathrm{EC}=$ electrical conductivity, $\mathrm{OM}=$ organic matter, $\mathrm{TC}=$ total carbon, $\mathrm{TN}=$ total nitrogen; $\mathrm{R}^{2}=$ coefficient of determination, $\mathrm{RMSEP}=$ root mean squared error of prediction, $\mathrm{SEP}=$ standard error of prediction. ${ }^{* 4}$ On DM basis. ${ }^{* 5} \mathrm{DM}$ calculated from parameter moisture $(\mathrm{m})$ by $\mathrm{DM}=1-\mathrm{m}$. 
Table 4. Summary of published data for predicting parameters in liquid organic manures based on optical spectroscopy. Additionally, publications about solid organic manures such as solid poultry manure or dairy faeces are included.

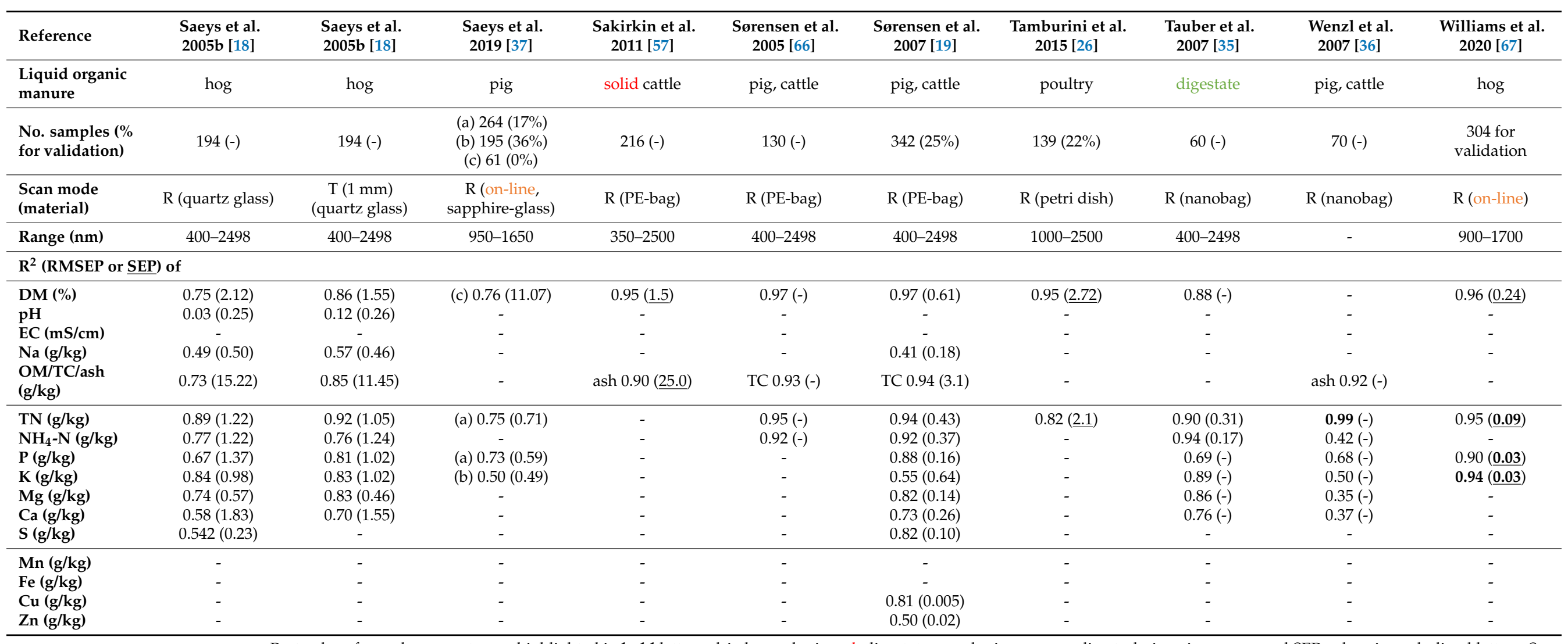

Best values for each parameter are highlighted in bold letters, dried samples in red, digestate samples in green, on-line technique in orange, and SEP values in underlined letters. Scan mode: $\mathrm{R}=$ reflectance $\mathrm{T}=$ transflectance; Parameters: $\mathrm{DM}=$ dry matter, $\mathrm{EC}=$ electrical conductivity, $\mathrm{OM}=$ organic matter, $\mathrm{TC}=$ total carbon, TN= total nitrogen, TDN = total dissolved nitrogen; $\mathrm{R}^{2}=$ coefficient of determination, RMSEP = root mean squared error of prediction, $\mathrm{SEP}=$ standard error of prediction. 
Table 5. Summary of published data for predicting parameters in liquid organic manures based on optical spectroscopy. Additionally, publications about solid organic manures such as solid poultry manure or dairy faeces are included.

\begin{tabular}{|c|c|c|c|c|c|c|c|c|c|c|}
\hline Reference & $\underset{\text { X68] }}{\text { Xing et al. } 2008}$ & $\begin{array}{c}\text { Yang et al. } 2006 \\
{[32]}\end{array}$ & $\begin{array}{c}\text { Ye et al. } 2005 \\
\text { [20] }\end{array}$ & $\begin{array}{c}\text { Ye et al. } 2005 \\
\text { [20] }\end{array}$ & $\begin{array}{c}\text { Ye et al. } 2005 \\
\text { [20] }\end{array}$ & $\begin{array}{c}\text { Ye et al. } 2005 \\
\text { [20] }\end{array}$ & $\begin{array}{c}\text { Ye et al. } 2005 \\
\text { [20] }\end{array}$ & $\begin{array}{c}\text { Ye et al. } 2005 \\
{[20]}\end{array}$ & $\begin{array}{l}\text { Zimmermann } \\
\text { et al. } 2008 \text { [69] }\end{array}$ & $\begin{array}{c}\text { Zimmermann } \\
\text { and Hartung } \\
2009[70]\end{array}$ \\
\hline $\begin{array}{l}\text { Liquid organic } \\
\text { manure }\end{array}$ & layer poultry & fattening pig & layer poultry & $\begin{array}{c}\text { poultry, broiler } \\
\text { litter }\end{array}$ & beef cattle & $\begin{array}{l}\text { swine solid } \\
\text { hoop }\end{array}$ & $\begin{array}{l}\text { swine liquid } \\
\text { lagoon }\end{array}$ & swine slurry & not specified & $\begin{array}{l}\text { DM: cattle, K: } \\
\text { mix, other: pig }\end{array}$ \\
\hline $\begin{array}{l}\text { No. samples (\% } \\
\text { for validation) }\end{array}$ & $91(-)$ & $108(33 \%)$ & $111(-)$ & $95(-)$ & $72(-)$ & $39(-)$ & $88(-)$ & $85(-)$ & $28(-)$ & $\begin{array}{c}\text { for DM: } 61(-), \\
\text { for K: } 149(-), \text { for } \\
\text { others: } 49(-)\end{array}$ \\
\hline $\begin{array}{l}\text { Scan mode } \\
\text { (material) }\end{array}$ & R (quartz-cup) & R (PE-bag) & R (PE-bag) & R (PE-bag) & R (PE-bag) & R (PE-bag) & R (PE-bag) & R (PE-bag) & $\begin{array}{c}\mathrm{R} \text { (on-line, } \\
\text { sapphire-glass) }\end{array}$ & $\begin{array}{c}\mathrm{R} \text { (on-line, } \\
\text { (sapphire-glass) }\end{array}$ \\
\hline Range (nm) & $1000-2500$ & $833-2500$ & $1100-2498$ & $1100-2498$ & $1100-2498$ & $1100-2498$ & $1100-2498$ & $1100-2498$ & $850-1650$ & $850-1650$ \\
\hline \multicolumn{11}{|c|}{$R^{2}$ (RMSEP or $\underline{\text { SEP) }}$ of } \\
\hline DM (\%) & $0.86(1.95)$ & $0.79 * 6(1.75)$ & $0.97(\underline{2.28})$ & $0.91(\underline{1.45})$ & $0.90(2.98)$ & $0.91(2.39)$ & $0.92(\underline{\mathbf{0 . 0 7}})$ & $0.92(\underline{0.68})$ & $0.98(0.27)$ & $0.95(0.54)$ \\
\hline $\mathrm{pH}$ & $0.55(0.15)$ & $0.50 * 6(0.25)$ & - & - & - & - & - & - & - & - \\
\hline $\mathrm{EC}(\mathrm{mS} / \mathrm{cm})$ & - & - & - & - & - & - & - & - & - & - \\
\hline $\mathrm{Na}(\mathrm{g} / \mathrm{kg})$ & $0.62(0.19)$ & - & $0.71(0.56)$ & $0.63(0.5)$ & $0.90(0.3)$ & $0.80(0.3)$ & $0.80(0.3)$ & $0.88(0.03)$ & - & - \\
\hline OM (g/kg) & $0.89(6.17)$ & $0.81 * 6(14.2)$ & - & - & - & - & - & - & - & - \\
\hline $\mathrm{TN}(\mathrm{g} / \mathrm{kg})$ & $0.88(1.13)$ & $0.56 * 6(1.44)$ & $0.92(2.3)$ & $0.80(1.3)$ & $0.88(1.1)$ & $0.87(0.7)$ & $0.83(1.2)$ & $0.91(0.21)$ & $0.96(0.25)$ & $0.96(0.29)$ \\
\hline $\mathrm{NH}_{4}-\mathrm{N}(\mathrm{g} / \mathrm{kg})$ & $0.88(0.86)$ & $0.84 * 6(0.40)$ & $0.91(\underline{0.26})$ & $0.89(\underline{0.31})$ & $0.89(0.2)$ & $0.92(0.4)$ & $0.88(\underline{1.0})$ & $0.91(\underline{0.14})$ & $0.98(0.11)$ & $0.94(0.22)$ \\
\hline$M g(g / k g)$ & $0.60(0.67)$ & $0.35 * 6(0.38)$ & $-\square$ & $-\square$ & - & - & - & - & - & - \\
\hline $\mathrm{Ca}(\mathrm{g} / \mathrm{kg})$ & - & - & $0.75(\underline{13.2})$ & $0.54(\underline{2.1})$ & $0.72(6.6)$ & $0.94(2.1)$ & $0.90(\underline{0.2})$ & $0.86(\underline{0.73})$ & - & - \\
\hline$S(g / k g)$ & - & - & $0.70(\underline{0.58})$ & $0.66(\underline{\underline{0.4}})$ & $0.78(0.4)$ & $0.70(0.56)$ & $0.83(\underline{\underline{0.1}})$ & $0.70(\underline{0.04})$ & - & - \\
\hline $\operatorname{Mn}(\mathrm{g} / \mathrm{kg})$ & - & - & - & - & - & - & - & - & - & - \\
\hline $\mathrm{Fe}(\mathrm{g} / \mathrm{kg})$ & - & - & - & - & - & - & - & - & - & - \\
\hline $\mathrm{Cu}(\mathrm{g} / \mathrm{kg})$ & $0.48(0.003)$ & $0.70 * 6(0.06)$ & $0.71(\underline{0.01})$ & $0.62(\underline{0.07})$ & $0.71(0.01)$ & $0.82(0.004)$ & - & $0.87(\underline{0.05})$ & - & - \\
\hline $\mathrm{Zn}(\mathrm{g} / \mathrm{kg})$ & $0.86(1.95)$ & $0.69 * 6(0.03)$ & $0.81(\overline{0.06})$ & $0.78(\underline{0.03})$ & $0.66(0.04)$ & $0.81(0.03)$ & $0.79(\underline{0.001)}$ & $0.83(\underline{0.01})$ & - & - \\
\hline
\end{tabular}

Best values for each parameter are highlighted in bold letters, dried samples in red, on-line technique in orange, and SEP values in underlined letters. Scan mode: $\mathrm{R}=$ reflectance Parameters: $\mathrm{DM}=$ dry matter, $\mathrm{EC}=$ electrical conductivity, $\mathrm{OM}=$ organic matter, $\mathrm{TN}=$ total nitrogen; $\mathrm{R}^{2}=$ coefficient of determination, $\mathrm{RMSEP}=$ root mean squared error of prediction, $\mathrm{SEP}=$ standard error of prediction. ${ }^{* 6}$ Calculated from RDP by $\mathrm{R}^{2}=1-\mathrm{RDP}^{-2}$ 
Table 6. Summary of published data displayed in Tables 1-5 describing parameters of liquid organic manures with optical spectroscopy.

\begin{tabular}{|c|c|c|c|c|c|}
\hline \multicolumn{2}{|c|}{ Parameter } & $\begin{array}{l}\text { Number of } \\
\text { Publications }\end{array}$ & Range of $\mathbf{R}^{2}$ & Range of RMSEP (SEP) & Reference of Best $\mathbf{R}^{2}$ (Best RMSEP or Best SEP) \\
\hline \multicolumn{2}{|c|}{ DM (\%) } & 40 & $0.58-0.99$ & $0.20-11.39(0.07-3.61)$ & Zimmermann et al. 2008 [69] (Ye et al. 2005 [20]) \\
\hline \multicolumn{2}{|c|}{$\mathrm{pH}$} & 11 & $0.03-0.96$ & $0.09-0.26(0.07-0.29)$ & Malley et al. 2001a [28] (Malley et al. 2001a [28]) \\
\hline \multicolumn{2}{|c|}{$\mathrm{EC}(\mathrm{mS} / \mathrm{cm})$} & 4 & $0.11-0.94$ & $1.41-2.55(0.77-1.17)$ & Malley et al. 2001a [28] (Malley et al. 2002 [14]) \\
\hline \multicolumn{2}{|c|}{$\mathrm{Na}(\mathrm{g} / \mathrm{kg})$} & 16 & $0.40-0.95$ & $0.04-0.50(0.03-0.56)$ & Malley et al. 2002 [14] (Malley et al. 2002 [14]) \\
\hline \multirow{4}{*}{$\begin{array}{c}\mathrm{C} \\
(\mathrm{g} / \mathrm{kg})\end{array}$} & $\mathrm{OM}$ & 8 & $0.57-0.93$ & $6.17-23.8$ (n.a.) & Du et al. 2010 [27] (Xing et al. 2008 [68]) \\
\hline & $\mathrm{TC}$ & 8 & $0.91-0.97$ & $3.1-14.5(2.90-23.4)$ & Asai et al. 1993 [61] (Althaus et al. 2013 [13]) \\
\hline & Susp. C & 2 & $0.98-0.99$ & n.a. $(0.7-1.56)$ & Malley et al. 2002 [14] (Malley et al. 2002 [14]) \\
\hline & ash & 5 & $0.81-0.99$ & $3.3-22.4(0.10-25.0)$ & Asai et al. 1993 [61] (Becaccia et al. 2015 [62]) \\
\hline \multirow{3}{*}{$\begin{array}{c}\mathrm{N} \\
(\mathrm{g} / \mathrm{kg})\end{array}$} & $\mathrm{TN}$ & 45 & $0.48-0.98$ & $0.16-8.94(0.09-2.3)$ & Wenzl et al. 2007 [36] (Williams et al. 2020 [67]) \\
\hline & $\mathrm{NH}_{4}-\mathrm{N}$ & 39 & $0.42-0.99$ & $0.08-3.87(0.07-1.0)$ & Zimmermann et al. 2008 [69] (Malley et al. 2002 [14]) \\
\hline & TDN & 2 & $0.94-0.95$ & n.a. $(0.14-0.20)$ & Malley et al. 2001a [28] (Malley et al. 2002 [14]) \\
\hline \multirow{2}{*}{$\begin{array}{c}\mathrm{P} \\
(\mathrm{g} / \mathrm{kg})\end{array}$} & $\mathrm{TP}$ & 38 & $0.47-0.99$ & $0.025-1.37(0.03-2.4)$ & Zimmermann et al. 2008 [69] (Zimmermann et al. 2008 [69]) \\
\hline & TDP & 1 & 0.99 & n.a. $(0.055)$ & Malley et al. 2002 [14] (Malley et al. 2002 [14]) \\
\hline \multicolumn{2}{|c|}{$\mathrm{K}(\mathrm{g} / \mathrm{kg})$} & 35 & $0.19-0.95$ & $0.04-1.4(0.03-2.9)$ & Williams et al. 2020 [67] (Williams et al. 2020 [67]) \\
\hline \multicolumn{2}{|c|}{$\mathrm{Mg}(\mathrm{g} / \mathrm{kg})$} & 17 & $0.35-0.98$ & $0.14-0.67(0.03-0.27)$ & Malley et al. 2002 [14] (Malley et al. 2002 [14]) \\
\hline \multicolumn{2}{|c|}{$\mathrm{Ca}(\mathrm{g} / \mathrm{kg})$} & 22 & $0.37-0.97$ & $0.26-6.6(0.025-13.2)$ & Malley et al. 2001a [28] (Malley et al. 2002 [14]) \\
\hline \multicolumn{2}{|c|}{$\mathrm{S}(\mathrm{g} / \mathrm{kg})$} & 17 & $0.36-0.95$ & $0.02-0.56(0.01-0.58)$ & Malley et al. 2001a [28] (Malley et al. 2001a [28]) \\
\hline \multicolumn{2}{|c|}{$\operatorname{Mn}(\mathrm{g} / \mathrm{kg})$} & 6 & $0.57-0.96$ & $0.009-0.07$ (0.005-0.01) & Malley et al. 2001a [28] (Malley et al. 2001c [30]) \\
\hline \multicolumn{2}{|c|}{$\mathrm{Fe}(\mathrm{g} / \mathrm{kg})$} & 4 & $0.77-0.97$ & $0.02-0.06(0.025-0.6)$ & Malley et al. 2001c [30] (Malley et al. 2001c [30]) \\
\hline \multicolumn{2}{|c|}{$\mathrm{Cu}(\mathrm{g} / \mathrm{kg})$} & 15 & $0.48-0.99$ & $0.003-0.055(0.005-0.07)$ & Malley et al. 2001a [28] (Xing et al. 2008 [68]) \\
\hline \multicolumn{2}{|c|}{$\mathrm{Zn}(\mathrm{g} / \mathrm{kg})$} & 16 & $0.12-0.98$ & $0.001-0.05(0.001-0.06)$ & Malley et al. 2001a [28] (Malley et al. 2001c [30]) \\
\hline
\end{tabular}

$\mathrm{DM}=$ dry matter, $\mathrm{EC}=$ electrical conductivity, $\mathrm{OM}=$ organic matter, $\mathrm{TC}=$ total carbon, $\mathrm{TOC}=$ total organic carbon, susp $\mathrm{C}=$ suspended carbon, $\mathrm{TN}=$ total nitrogen, $\mathrm{TDN}=$ total dissolved nitrogen, $\mathrm{TP}=$ total phosphorus, TDP $=$ total dissolved phosphorus; n.a.= not available

Asai et al. [61] were probably the first to publish data using NIR spectrometry to estimate nutrients in livestock manures. Their values indicating the quality of regressions for crude ash $\left(\mathrm{R}^{2}=0.99, \mathrm{SEP}=22.4 \mathrm{~g} / \mathrm{kg}\right)$ and total carbon $(\mathrm{TC})\left(\mathrm{R}^{2}=0.97, \mathrm{SEP}=14.5 \mathrm{~g} / \mathrm{kg}\right)$ in dried cattle manures are still the best results for these parameters gained to the present day. Twenty years later, Althaus et al. [13] obtained a similarly good $\mathrm{R}^{2}$ of 0.96 for TC for dried dairy manures, but with a reasonably better result for SEP of $2.90 \mathrm{~g} / \mathrm{kg}$. Du et al. [27] used a MIR spectrometer with a photoacoustic measuring mode to obtain the best results for organic matter (OM) of liquid manures (pig, poultry, and cattle; $\mathrm{R}^{2}=0.93, \mathrm{RMSEP}=23.8 \mathrm{~g} / \mathrm{kg}$ ).

Malley et al. [14] obtained highest values in transflection mode for suspended $C\left(R^{2}=0.99\right.$, $\mathrm{SEP}=0.7 \mathrm{~g} / \mathrm{kg})$, total dissolved $\mathrm{P}\left(\mathrm{R}^{2}=0.99, \mathrm{SEP}=0.055 \mathrm{~g} / \mathrm{kg}\right), \mathrm{Na}\left(\mathrm{R}^{2}=0.95, \mathrm{SEP}=0.03 \mathrm{~g} / \mathrm{kg}\right)$, and $\mathrm{Mg}\left(\mathrm{R}^{2}=0.98, \mathrm{SEP}=0.03 \mathrm{~g} / \mathrm{kg}\right)$. Malley et al. [28] best predicted EC with $\mathrm{R}^{2}=0.94$ and $\mathrm{SEP}=1.17 \mathrm{~g} / \mathrm{kg}$ and $\mathrm{pH}$ with $\mathrm{R}^{2}=0.96$ and $\mathrm{SEP}=0.07 \mathrm{~g} / \mathrm{kg}$. The reason why they obtained such a good $\mathrm{R}^{2}$ for the $\mathrm{pH}$ value is not clear, since other studies could not confirm this high correlation with $\mathrm{pH}$ (e.g., [18,24,32,33,68]). Malley et al. [30] published very good values for several plant nutrients (i.e., for $\mathrm{S} \mathrm{R}^{2}=0.95, \mathrm{SEP}=0.01 \mathrm{~g} / \mathrm{kg}$; for $\mathrm{Ca} \mathrm{R}^{2}=0.97, \mathrm{SEP}=0.21 \mathrm{~g} / \mathrm{kg}$; for $\mathrm{Mn} \mathrm{R}^{2}=0.96, \mathrm{SEP}=0.005 \mathrm{~g} / \mathrm{kg}$; for Fe $\mathrm{R}^{2}=0.96, \mathrm{SEP}=0.025 \mathrm{~g} / \mathrm{kg}$; for Cu $\mathrm{R}^{2}=0.99$,

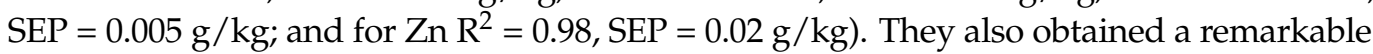
high correlation for $\mathrm{Na}\left(\mathrm{R}^{2}=0.85\right)$, even though it was not correlated with any other constituents. Thus, they concluded that the solved $\mathrm{Na}$, which is supposed to have no optically active properties, probably influenced the reflecting spectrum of water including its hydration shells. Consequently, they suspected solved $\mathrm{Na}$ to be indirectly detected. This optophysical mechanism has to be kept in mind also for other soluble nutrients (e.g., $\mathrm{K}^{+}$).

Best results for the main plant nutrients nitrogen and phosphorus in liquid livestock manures were published by Zimmermann et al. [69] for TN $\left(\mathrm{R}^{2}=0.96, \mathrm{RMSEP}=0.25 \mathrm{~g} / \mathrm{kg}\right)$, for $\mathrm{NH}_{4}-\mathrm{N}\left(\mathrm{R}^{2}=0.98, \mathrm{RMSEP}=0.11 \mathrm{~g} / \mathrm{kg}\right)$, and for $\mathrm{P}\left(\mathrm{R}^{2}=0.98, \mathrm{RMSEP}=0.025 \mathrm{~g} / \mathrm{kg}\right)$. They also achieved the highest correlations for dry matter content (DM) with $\mathrm{R}^{2}=0.98$ and $\mathrm{RMSEP}=0.27 \mathrm{~g} / \mathrm{kg}$. They used a diode array spectrometer combined with two simultaneously measuring sensors, fixed on a slurry tanker behind two sapphire windows enabling 
on-line and real-time measurements during slurry application in the field. Nevertheless, based on a larger sample set, they published lower values at the end of their study, especially for $\mathrm{P}$ $\left(\mathrm{R}^{2}=0.86\right.$; [70]; Table 5). Williams et al. [67] published best correlation values for $\mathrm{K}\left(\mathrm{R}^{2}=0.94\right.$, $\mathrm{SEP}=0.03 \mathrm{~g} / \mathrm{kg})$, using 304 samples of lagoon hog manure for the validation of their on-line NIRS system using four equal spectrometers in a line.

The question of which parameters of the whole measurement process can be recommended to obtain the best prediction results cannot be answered easily because the complex setups of the examined studies are not always comparable with each other. Nevertheless, it can be stated that an intensive homogenization is needed for a representative sampling or subsampling and additionally immediately before starting the spectroscopic measurements. To avoid the need for homogenization, drying might be an option. However, drying is an additional time-consuming work step that even seems to impair the prediction performance [16,22,34]. Finzi et al. [25] tested filtering as an alternative way of obtaining homogeneous samples. Although this pretreatment step improved prediction accuracy, their procedure might not be advisable because they only correlated the spectra of the eluates to the lab reference eluate concentrations. Thus, the true nutrient concentrations of the raw manures remain unknown because it can be assumed that nutrient concentrations in raw manures are just partly correlated to those of the eluates or the filter residues.

Cabassi et al. [21] showed that under laboratory conditions benchtop spectrometers with a wide wavelength range and a high resolution lead to better results compared to miniaturized portable spectrometers with a lower wavelength range and resolution.

However, it is expected that in the future such low-cost spectrometers can lead to similarly good results [71] because of technical, statistical, and procedural improvements. Moreover, it can be summarized that the VIS region does not seem to provide much more additional information than the NIR region, and thus VIS wavelengths might be negligible. Comparing NIR to MIR, the MIR technique has been scarcely used to assess nutrient concentrations in liquid organic fertilizers. Up to now, only Kemsley et al. [12], Reeves [16], and Du et al. [27] published articles applying MIR spectrometry for the characterization of animal manures. However, a clear preference for either NIR or MIR spectroscopy was not shown, but the fact that MIR spectrometers are in general more expensive than NIR instruments might be an argument to prefer NIR spectrometry.

According to Malley et al. [29] and Saeys et al. [18], the transflection measuring mode leads to more accurate calibrations in comparison with the reflection mode. However, the application of the transflection mode is more challenging because it requires a very thin sample layer of 1-2 $\mathrm{mm}$ in front of the sensor head. The heterogeneity of liquid organic manures with some particles larger than $2 \mathrm{~mm}$ and the corresponding difficulty of achieving a homogeneous distribution of the sample in the small gap between the reflector and the glass surface make transflection mode unsuitable, especially in the context of on-line applications [18].

A further aspect that needs to be addressed is the material for sample vessels. The less light is absorbed by this material, the more intensive signals with less noisy sample spectra can be detected. For this reason, sapphire glass is superior to quartz glass whereas quartz glass is superior to ordinary borosilicate glass. Polyethylene (PE) bags have the advantage of not breaking like glass, and the liquid slurry inside the bags can more easily be mixed without splashing. This is probably the reason why PE bags had been utilized in many of the presented studies. However, PE bags are not useful for continual measurements, which are needed for on-line applications. There, sapphire glass is the best option.

At least one of the classical preprocessing methods such as SG1, SG2, SNV, and MSC or combinations of them were utilized in almost all the presented studies. Although no preprocessing stood out with better results, it was shown that using at least one preprocessing method generally improved prediction performance (e.g., [32]). Furthermore, more sophisticated preprocessing methods such as DOSC may, in some cases, be superior to other preprocessing methods, as was shown by Chen et al. [45] for predicting N, P, and $\mathrm{K}$ by poultry manure spectra. 
Regarding the best suitable regression model, PLSR seems to be the favorite one, as this algorithm was used by most of the researchers. However, from the present state of research, it cannot be assessed if more sophisticated machine learning methods such as LASSO, random forest, or ANNs are superior to PLSR because there are almost no data available in that context. Only Chen et al. [51] compared ANNs with PLSR using spectra of poultry manures and found an overall improvement for $\mathrm{TN}, \mathrm{NH}_{4}-\mathrm{N}, \mathrm{P}$, and $\mathrm{K}$ by using ANNs.

The question of if it is better to use separate calibration models for pig, cattle, digestate, mixed manure, etc., or if it makes more sense to use a global calibration model for all types of manure and digestate cannot be answered based on the presented studies. However, it is supposed to use an adapted model for each manure type, as they have their own characteristics despite their great similarities.

Saeys et al. [37], Williams et al. [67], Zimmermann et al. [69], and Zimmermann and Hartung [70] demonstrated that it is possible to transfer NIR spectrometry from laboratory to on-line applications using NIR spectrometers mounted on slurry tankers. In particular, the published calibration $\mathrm{R}^{2} \mathrm{~s}$ of Zimmermann and Hartung [70] in the range of 0.86 to 0.96 and the validation $\mathrm{R}^{2} \mathrm{~s}$ of Williams et al. [67] in the range of 0.90 to 0.96 for the parameters $\mathrm{N}$, $\mathrm{P}, \mathrm{K}$, and DM are very promising for on-line applications at farm level. When such on-farm NIR systems are used for measuring nutrient concentrations in liquid organic manures, a changing ambient temperature might influence the reliability of the data. However, Finzi et al. [25] came to the conclusion that temperatures varying between 10 and $35{ }^{\circ} \mathrm{C}$ have no significant impact on the results. However, the effect of colder temperatures between 0 and $10{ }^{\circ} \mathrm{C}$ has not been examined.

\section{Applications in Laboratory and Field Practice}

In recent years, there has been intensive progress in commercializing and formalizing the NIR technique for determining nutrient concentrations and physicochemical parameters in livestock manures and biogas digestates.

The commercial lab Eurofins Agro (Wageningen, the Netherlands) offers NIR measurements as an alternative to wet chemical standard methods for the analysis of manures and biosolids. Besides $\mathrm{TN}, \mathrm{NH}_{4}-\mathrm{N}, \mathrm{P}$, and $\mathrm{K}, \mathrm{Mg}$ and $\mathrm{S}$ are also determined using NIR spectrometry.

In cooperation with LFZ Raumberg-Gumpenstein (Irdning, Austria), IPUS Mineral\& Umwelttechnologie GmbH (Rottenmann, Austria) developed a laboratory service for liquid organic manure analysis focusing on $\mathrm{DM}$, ash, $\mathrm{pH}, \mathrm{TN}, \mathrm{NH}_{4}-\mathrm{N}, \mathrm{P}, \mathrm{K}, \mathrm{Ca}$, and $\mathrm{Mg}$, as well as urea and volatile fatty acids. As sample containers, they are using so-called Nanobags $[35,36]$, which are filled with a strongly absorbing carrier material based on zeolites. Their VIS-NIR measurements are cheaper and faster in comparison to wet chemical standard analysis.

Carl Zeiss AG (Oberkochen, Germany) sells the NIR spectrometer "Corona extreme" to measure several parameters in various agricultural products, including manure. This device is also supposed to be installable on agricultural vehicles (e.g., slurry tankers) for online measurements of $\mathrm{TN}, \mathrm{NH}_{4}-\mathrm{N}, \mathrm{P}$, and $\mathrm{K}$. In cooperation with Carl Zeiss AG, the globally acting agricultural company John Deere (Moline, IL, USA) developed the measurement system HarvestLab ${ }^{\mathrm{TM}}$ (John Deere, Moline, IL, USA) for NIR on-line and on-site analysis of agricultural products (e.g., maize silage, liquid organic manures). With the support of the Belgian company Joskin SA (Soumagne, Belgium), this device was mounted to the flow path of slurry tankers to enable real-time analysis of $\mathrm{DM}, \mathrm{TN}, \mathrm{NH}_{4}-\mathrm{N}, \mathrm{P}$, and $\mathrm{K}$ in liquid organic manures during field application. For this method, the German subsidiary company John Deere GmbH \& Co. KG (Walldorf, Germany) received a certification seal by the German Agricultural Society (DLG) (Frankfurt am Main, Germany) for TS, TN, NH $4-\mathrm{N}_{\text {, }}$ $\mathrm{P}$, and $\mathrm{K}$ in cattle manures, pig manures, and digestates (Table 7). The certificate assures that at least $60 \%$ of the measured samples differ less than $25 \%$ and no sample differs more than $35 \%$ from the reference lab value [72]. The cooperating German companies m-u-t $\mathrm{GmbH}$ (Wedel, Germany) and Zunhammer GmbH (Traunreut, Germany) also received a certification by the DLG for their on-line and real-time NIRS system to determine TS, 
$\mathrm{TN}, \mathrm{NH}_{4}-\mathrm{N}, \mathrm{P}$, and $\mathrm{K}$ in cattle manures, pig manures, and digestates. This system was also the first NIRS system that was certified by DLG for analysing mixed pig and cattle manures. This NIRS system is also utilized by the companies Kotte Landtechnik $\mathrm{GmbH}$ (Rieste, Germany), Kamps de Wild and Kaweco (Winkelskamp, the Netherlands), Veenhuis Machines (Rijssen, the Netherlands), and Topcon Agriculture (Madrid, Spain); however, each company received its own certificate (Table 7). In addition, the Italian Dinamica Generale S.p.A.( Poggio Rusco, Italy) received a certificate for their on-line system to analyse TN in liquid pig manure [73]. A German start-up company (TENIRS GmbH, Kiel, Germany) tried to commercialize on-line NIR analyses of nutrients in liquid manures and biogas substrates. However, their innovative technique using a special flow-through cell in bypass mode [74] could not establish on the market. The company was later bought by m-u-t GmbH.

The next step for an established on-field application is to embed the NIRS technology for liquid organic manures in the law. Presently, this technology is officially approved in the Netherlands as well as 3 out of 16 German federal states, although a quality assurance system is still missing to guarantee sufficient accuracy over time. However, the developments of such quality management standards are in progress [75].

Table 7. Commercial NIR sensors, certified by the German DLG.

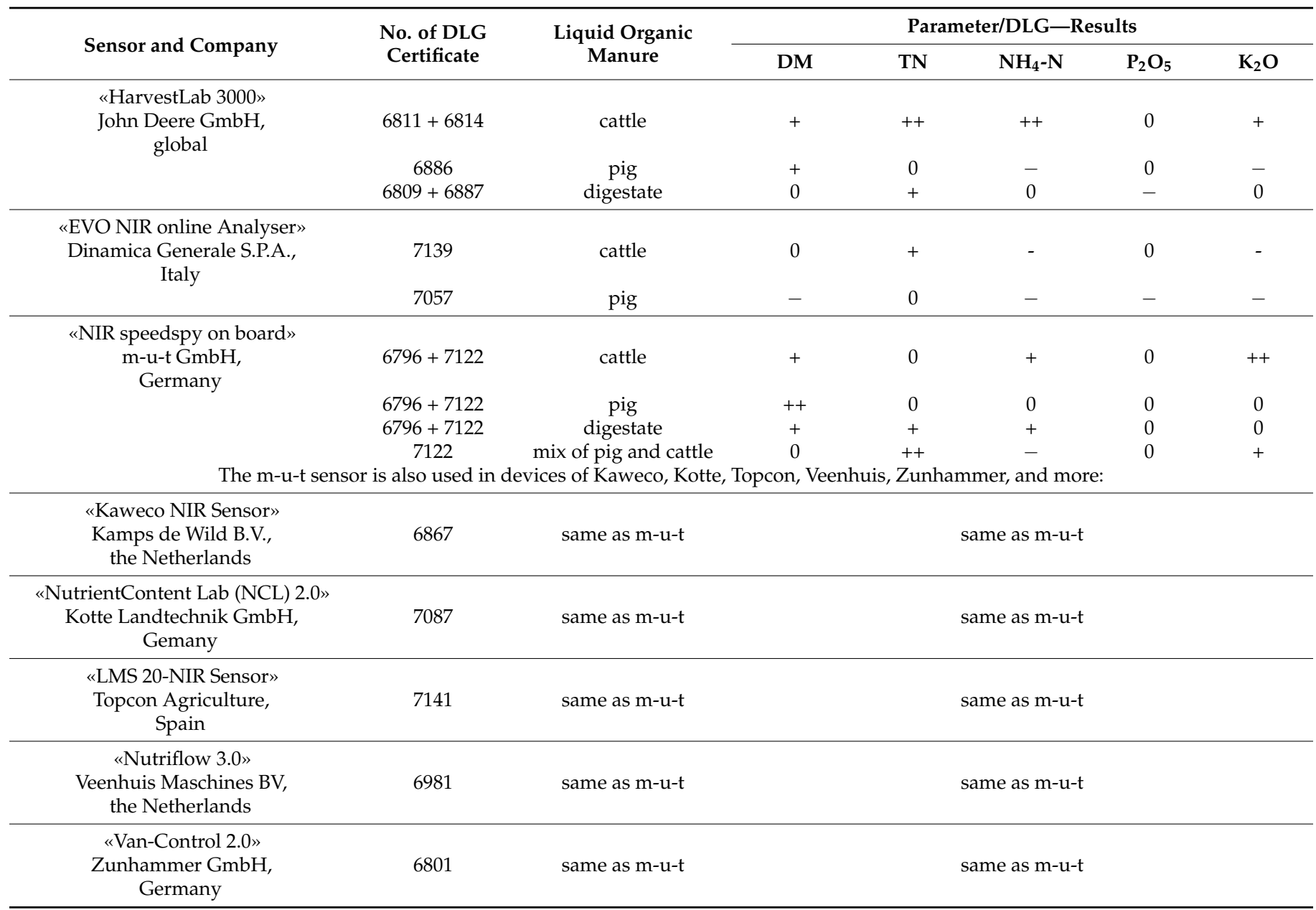

$++: 4 / 5$ of the tested sample concentrations differ less than $10 \%$ and no sample concentration more than $20 \%$ from the reference; $+: 4 / 5$ of the tested sample concentrations differ less than $15 \%$ and no sample concentration more than $25 \%$ from the reference; $0: 3 / 5$ of the tested sample concentrations differ less than $25 \%$ and no sample concentration more than $35 \%$ from the reference; - : not passed or not tested. 


\section{Conclusions}

This literature survey on using optical spectroscopy to analyse physicochemical parameters and macro- and micronutrient concentrations in liquid organic manures (for reviewing information about further organic fertilizers such as composts, the reader is referred to [76]) reveals a great potential for NIRS to be established as an alternative measurement method, be it for manure application or manure selling purposes. Best prediction models could at least achieve a determination coefficient of 0.93 for each mentioned parameter. However, it has to be kept in mind that almost all presented papers focused on laboratory setups. The assumption that dynamic on-line measurements at the farm level can continue this successful progress in characterizing liquid organic manures (e.g., during the filling operation of slurry tankers or during field application) could be confirmed by a few studies and rather new developments of some agricultural companies. They now offer such systems for on-line measurements and site-specific manure applications and give this technology the possibility to manifest in agribusiness.

However, despite the obvious progress in optical spectroscopy over the last 20 years, there is still potential to optimize this method to (1) improve predictions by sophisticated chemometric methods, e.g., machine learning algorithms; (2) assure sufficient accuracy by good quality management with regularly recurring calibrations; and (3) reduce costs, e.g., by using cheaper spectrometers. All these aspects are important to establish optical spectroscopy (mainly NIRS) as an alternative technology permitted by law to analyse liquid organic manures with the final aim of precisely applying these fertilizers for optimizing agricultural yield and reducing environmental pollution.

Author Contributions: Conceptualization, M.H., R.G. and H.-W.O.; formal analysis, M.H.; investigation, M.H.; data curation, M.H.; writing-original draft preparation, M.H.; writing-review and editing, S.V., H.D. and H.-W.O.; visualization, M.H.; supervision, R.G. and S.V.; project administration, R.G., S.V. and H.-W.O.; funding acquisition, R.G., S.V. and H.-W.O. All authors have read and agreed to the published version of the manuscript.

Funding: This research was funded by the German Federal Environmental Foundation (Deutsche Bundesstiftung Umwelt-DBU), grant No. AZ 33702.

Conflicts of Interest: The authors declare no conflict of interest.

\section{References}

1. Zhang, H.; Dao, T.; Basta, N.; Dayton, E.; Daniel, T. Remediation techniques for manure nutrient loaded soils. In Animal Agriculture and the Environment-National Center for Manure \& Animal Waste Management White Paper; American Society of Agricultural and Biological Engineers (ASABE): St. Joseph, MI, USA, 2006.

2. Lin, Y.; Ye, G.; Kuzyakov, Y.; Liu, D.; Fan, J.; Ding, W. Long-term manure application increases soil organic matter and aggregation, and alters microbial community structure and keystone taxa. Soil Biol. Biochem. 2019, 134, 187-196. [CrossRef]

3. Withers, P.J.A.; Haygarth, P.M. Agriculture, phosphorus and eutrophication: A European perspective. Soil Use Manag. 2007, 23, 1-4. [CrossRef]

4. Zhu, J.; Ndegwa, P.M.; Zhang, Z. Manure sampling procedures and nutrient estimation by the hydrometer method for gestation pigs. Bioresour. Technol. 2004, 92, 243-250. [CrossRef] [PubMed]

5. Saeys, W. Technical Tools for the Optimal Use of Animal Manure as a Fertiliser: On-Line Composition Measurement and Manure Injection Control. Ph.D. Thesis, Katholieke Universiteit Leuven, Leuven, Belgium, 2006.

6. Rubenschuh, U.; Volz, F. Nährstoffgehalte in Gülle Online Mit Sensoren Bestimmen; DLG kompakt, No. 8; DLG e.V: Frankfurt am Main, Germany, 2019.

7. Burns, D.A.; Ciurczak, E.W. Handbook of Near-Infrared Analysis, 3rd ed.; CRC Press: Boca Raton, FL, USA, 2007.

8. Schmidt, W. Optical Spectroscopy in Chemistry and Life Sciences; Wiley-VCH: Weinheim, Germany, 2005; ISBN 3-527-29911-4.

9. Suzuki, T.; Nakagawa, T.; Ohkubo, K.; Fukuzumi, S.; Matsuo, Y. Electronic infrared light absorption of a tri-palladium complex containing two $\pi$-expanded tetracene ligands. Chem. Sci. 2014, 5, 4888-4894. [CrossRef]

10. Shenk, J.S.; Workman, J.J.; Westerhaus, M.O. Application of NIR spectroscopy to agricultural products. In Handbook of Near-Infrared Analysis, 3rd ed.; Burns, D.A., Ciurczak, E.W., Eds.; CRC Press: Boca Raton, FL, USA, 2007; pp. 347-383. ISBN 978-0-4291-2301-6.

11. Saeys, W.; Mouazen, A.M.; Ramon, H. Potential for onsite and online analysis of pig manure using visible and near infrared reflectance spectroscopy. Biosyst. Eng. 2005, 91, 393-402. [CrossRef] 
12. Kemsley, E.K.; Tapp, H.S.; Scarlett, A.J.; Miles, S.J.; Hammond, R.; Wilson, R.H. Comparison of spectroscopic techniques for the determination of Kjeldahl and ammoniacal nitrogen content of farmyard manure. J. Agric. Food Chem. 2001, 49, 603-609. [CrossRef]

13. Althaus, B.; Papke, G.; Sundrum, A. Technical note: Use of near infrared reflectance spectroscopy to assess nitrogen and carbon fractions in dairy cow feces. Anim. Feed Sci. Technol. 2013, 185, 53-59. [CrossRef]

14. Malley, D.F.; Yesmin, L.; Eilers, R.G. Rapid analysis of hog manure and manure-amended soils using near-infrared spectroscopy. Soil Sci. Soc. Am. J. 2002, 66, 1677. [CrossRef]

15. Millmier, A.; Lorimor, J.; Hurburgh, C., Jr.; Fulhage, C.; Hattey, J.; Zhang, H. Near-infrared sensing of manure nutrients. Trans. Am. Soc. Agric. Biol. Eng. (ASAE) 2000, 43, 903-908. [CrossRef]

16. Reeves, J.B. Near- versus mid-infrared diffuse reflectance spectroscopy for determination of minerals in dried poultry manure. Poult. Sci. 2001, 80, 1437-1443. [CrossRef]

17. Reeves, J.B. Near-infrared diffuse reflectance spectroscopy for the analysis of poultry manures. J. Agric. Food Chem. 2001, 49, $2193-2197$. [CrossRef] [PubMed]

18. Saeys, W.; Xing, J.; de Baerdemaeker, J.; Ramon, H. Comparison of transflectance and reflectance to analyse hog manures. J. Near Infrared Spectrosc. 2005, 13, 99-107. [CrossRef]

19. Sørensen, L.K.; Sørensen, P.; Birkmose, T.S. Application of reflectance near infrared spectroscopy for animal slurry analyses. Soil Sci. Soc. Am. J. 2007, 71, 1398. [CrossRef]

20. Ye, W.; Lorimor, J.C.; Hurburgh, C.; Zhang, H.; Hattey, J. Application of near-infrared reflectance spectroscopy for determination of nutrient contents in liquid and solid manures. Trans. Am. Soc. Agric. Biol. Eng. (ASAE) 2005, 48, 1911-1918. [CrossRef]

21. Cabassi, G.; Cavalli, D.; Fuccella, R.; Marino Gallina, P. Evaluation of four NIR spectrometers in the analysis of cattle slurry. Biosyst. Eng. 2015, 133, 1-13. [CrossRef]

22. Malley, D.F.; McClure, C.; Martin, P.D.; Buckley, K.; McCaughey, W.P. Compositional analysis of cattle manure during composting using a field-portable near-infrared spectrometer. Commun. Soil Sci. Plant Anal. 2005, 36, 455-475. [CrossRef]

23. Mouazen, A.M.; Saeys, W.; Xing, J.; de Baerdemaeker, J.; Ramon, H. Near infrared spectroscopy for agricultural materials: An instrument comparison. J. Near Infrared Spectrosc. 2005, 13, 87-97. [CrossRef]

24. Saeys, W.; Darius, P.; Ramon, H. Potential for on-site analysis of hog manure using a visual and near infrared diode array reflectance spectrometer. J. Near Infrared Spectrosc. 2004, 12, 299-309. [CrossRef]

25. Finzi, A.; Oberti, R.; Negri, A.S.; Perazzolo, F.; Cocolo, G.; Tambone, F.; Cabassi, G.; Provolo, G. Effects of measurement technique and sample preparation on NIR spectroscopy analysis of livestock slurry and digestates. Biosyst. Eng. 2015, 134, 42-54. [CrossRef]

26. Tamburini, E.; Castaldelli, G.; Ferrari, G.; Marchetti, M.G.; Pedrini, P.; Aschonitis, V.G. Onsite and online FT-NIR spectroscopy for the estimation of total nitrogen and moisture content in poultry manure. Environ. Technol. 2015, 36, 2285-2294. [CrossRef]

27. Du, C.; Zhou, G.; Jianmin, Z.; Huoyan, W.; Xiaoqin, C.; Yuanhua, D.; Hui, W. Characterization of animal manures using mid-infrared photoacoustic spectroscopy. Bioresour. Technol. 2010, 101, 6273-6277. [CrossRef]

28. Malley, D.; Martin, P.; Woods, S.E. Analysis of Nutrients in Hog Manure by Field-Portable Near-Infrared Spectroscopy: Development of a Mobile Laboratory and Results for Foss NIR Systems Inc. Model 6500 Spectrophotometer in the Laboratory: Final Report 1 of 3 to CETAC-West on Manure Demo Project; PDK Projects, Inc.: Nanaimo, BC, Canada, 2001.

29. Malley, D.; Martin, P.; Moffatt, T. Analysis of Minor Elements and Metals in Hog Manure by Field-Portable NIR Spectroscopy: Results for the Textron-Case NH Pro Spectra-Spectrometer: Final Report 2 of 3 to Manitoba Livestock Manure Management Initiative on Project 00-02-03; PDK Projects, Inc.: Nanaimo, BC, Canada, 2001.

30. Malley, D.; Martin, P.; Dettman, L. Analysis of Minor Elements and Metals in Hog Manure by Field-Portable Near-Infrared Spectroscopy: Results for the Zeiss Corona ${ }^{\circledR S p e c t r o m e t e r: ~ F i n a l ~ R e p o r t ~} 3$ of 3 to Manitoba Livestock Manure Management Initiative on Project 00-02-03; PDK Projects, Inc.: Nanaimo, BC, Canada, 2001.

31. Bries, J.; Vanongeval, L.; Goppens, G. Variaties in Samenstelling van Dierlijke Mest: Bë̈nvloedende Factoren en te nemen Voorzoren voor Verantwoord Gebruik Als Meststof; KVIV Studiedag Mestproblematiek: Antwerp, Belgium, 1997.

32. Yang, Z.; Han, L.; Fan, X. Rapidly Estimating Nutrient Contents of Fattening Pig Manure from Floor Scrapings by near Infrared Reflectance Spectroscopy. J. Near Infrared Spectrosc. 2006, 14, 261-268. [CrossRef]

33. De Ferrari, G.; Gallina, P.M.; Cabassi, G.; Bechini, L.; Maggiore, T. Near infrared spectral analysis of cattle slurries from Lombardy (Northern Italy) breeding farms NIR. In NIR 2005- NIR in Action. Making a Difference. Near Infrared Spectroscopy Proceedings of the 12th International Conference, Auckland, New Zealand, 9-15 April 2005; NZNIRSS-The New Zealand Near Infrared Spectroscopy Society: Auckland, New Zealand, 2005.

34. Dong, Y.; Chen, Y.; Zhu, D.; Li, Y.; Xu, C.; Bai, W.; Wang, Y.; Li, Q. The determination of total N, total P, Cu and Zn in chicken manure using near infrared reflectance spectroscopy. In Computer and Computing Technologies in Agriculture IV.; Li, D., Liu, Y., Chen, Y., Eds.; Springer: Berlin/Heidelberg, Germany, 2011; pp. 92-98. ISBN 978-3-642-18353-9.

35. Tauber, M.; Wenzl, W.; Somitsch, W. NIRS-Nanobag Methode als Schnellanalytik von Gärgut für die Begleitende Prozesskontrolle von Landwirtschaftlichen Biogasanlagen. In Gute Herstellungspraxis für Pflanzliche Produkte; ALVA Tagungsbericht: Wien, Austria, 2007; pp. 30-32.

36. Wenzl, W.; Steiner, B.; Haberl, L. Feldstudie zur NIRS-Gülleanalyse und Vergleich der Nährstoffbilanzen mit Tabellenwerten. In Gute Herstellungspraxis für Pflanzliche Produkte; ALVA Tagungsbericht: Wien, Austria, 2007; pp. 33-34. 
37. Saeys, W.; Watté, R.; Postelmans, A. Role of NIR Spectroscopy in Precision Fertilisation with Manure; International Fertilizer Society: Colchester, UK, 2019; pp. 1-21.

38. Arnold, M.A.; Small, G.W. Determination of physiological levels of glucose in an aqueous matrix with digitally filtered Fourier transform near-infrared spectra. Anal. Chem. 1990, 62, 1457-1464. [CrossRef]

39. Savitzky, A.; Golay, M.J.E. Smoothing and Differentiation of Data by Simplified Least Squares Procedures. Anal. Chem. 1964, 36, 1627-1639. [CrossRef]

40. Barnes, R.J.; Dhanoa, M.S.; Lister, S.J. Standard normal variate transformation and de-trending of near-infrared diffuse reflectance spectra. Appl. Spectrosc. 1989, 43, 772-777. [CrossRef]

41. Martens, H.; Jensen, S.A.; Geladi, P. Martens; Harald; Jensen, S.A.; Geladi, P. Multivariate linearity transformation for near-infrared reflectance spectrometry. In Proceedings of the Nordic Symposium on Applied Statistics, Stavanger, Norway, 12-14 June 1983.

42. Martens, H.; Stark, E. Extended multiplicative signal correction and spectral interference subtraction: New preprocessing methods for near infrared spectroscopy. J. Pharm. Biomed. Anal. 1991, 9, 625-635. [CrossRef]

43. Wold, S.; Antti, H.; Lindgren, F.; Öhman, J. Orthogonal signal correction of near-infrared spectra. Chemom. Intell. Lab. Syst. 1998, 44, 175-185. [CrossRef]

44. Westerhuis, J.A.; de Jong, S.; Smilde, A.K. Direct orthogonal signal correction. Chemom. Intell. Lab. Syst. 2001, 56, 13-25. [CrossRef]

45. Chen, L.J.; Xing, L.; Han, L.J. Influence of data preprocessing on the quantitative determination of nutrient content in poultry manure by near infrared spectroscopy. J. Environ. Qual. 2010, 39, 1841. [CrossRef]

46. Wold, H. Soft Modelling by Latent Variables: The Non-Linear Iterative Partial Least Squares (NIPALS) Approach. J. Appl. Probab. 1975, 12, 117-142. [CrossRef]

47. Mevik, B.-H.; Wehrens, R. The pls package: Principal component and partial least squares regression in R. J. Stat. Softw. 2007, 18, 1-23. [CrossRef]

48. Sternberg, J.C.; Stillo, H.S.; Schwendeman, R.H. Spectrophotometric analysis of multicomponent systems using least squares method in matrix form. Ergosterol irradiation system. Anal. Chem. 1960, 32, 84-90. [CrossRef]

49. Dagnew, M.; Crowe, T.; Schoenau, J. Measurement of nutrients in Saskatchewan hog manures using near-infrared spectroscopy. Can. Biosyst. Eng. 2004, 46, 33-37.

50. Malley, D.F.; Currie, R.S. Feasibility Study on the Rapid Analysis of Available N and P in Hog Manure and Manure-Amended Soils by Near-Infrared Spectroscopy: Report; PDK Projects, Inc.: Nanaimo, BC, Canada, 1999.

51. Chen, L.J.; Xing, L.; Han, L.J. Quantitative determination of nutrient content in poultry manure by near infrared spectroscopy based on artificial neural networks. Poult. Sci. 2009, 88, 2496-2503. [CrossRef] [PubMed]

52. Tibshirani, R. Regression shrinkage and selection via the lasso. J. R. Stat. Society. Ser. B 1996, 58, 267-288. [CrossRef]

53. Hoerl, A.E.; Kennard, R.W. Ridge regression: Applications to nonorthogonal problems. Technometrics 1970, 12, 69-82. [CrossRef]

54. Efron, B.; Hastie, T.; Johnstone, I.; Tibshirani, R. Least angle regression. Ann. Stat. 2004, 32, 407-499. [CrossRef]

55. Breiman, L. Random Forests. Mach. Learn. 2001, 45, 5-32. [CrossRef]

56. Reeves, J.B.; van Kessel, J.S. Near-infrared spectroscopic determination of carbon, total nitrogen, and ammonium-N in dairy manures. J. Dairy Sci. 2000, 83, 1829-1836. [CrossRef]

57. Sakirkin, S.L.P.; Morgan, C.L.S.; MacDonald, J.C.; Auvermann, B.W. Effect of diet composition on the determination of ash and moisture content in solid cattle manure using visible and near-infrared spectroscopy. Appl. Spectrosc. 2011, 65, 1056-1061. [CrossRef]

58. Williams, P.C. Interpretation of statistical evaluation of NIR analysis. In Near-Infrared Technology in the Agriculture and Food Industries; American Association of Cereal Chemists, Inc.: St. Paul, MN, USA, 1987; pp. 146-148. [CrossRef]

59. Minasny, B.; McBratney, A. Why you don't need to use RPD. Pedometron 2013, 33, 14-15.

60. Bellon-Maurel, V.; Fernandez-Ahumada, E.; Palagos, B.; Roger, J.-M.; McBratney, A. Critical review of chemometric indicators commonly used for assessing the quality of the prediction of soil attributes by NIR spectroscopy. Trends Anal. Chem. (TrAC) 2010, 29, 1073-1081. [CrossRef]

61. Asai, T.; Shimizu, S.; Koga, T.; Sato, M. Quick determination of total nitrogen, total carbon and crude ash in cattle manure using near infrared reflectance spectroscopy. Jpn. J. Soil Sci. Plant Nutr. 1993, 64, 669-675. [CrossRef]

62. Becaccia, A.; Ferrer, P.; Ibañez, M.A.; Estellés, F.; Rodríguez, C.; Moset, V.; de Blas, C.; Calvet, S.; García-Rebollar, P. Relationships among slurry characteristics and gaseous emissions at different types of commercial Spanish pig farms. Spanish J. Agric. Res. 2015, 13, e0602. [CrossRef]

63. Benozzo, F.; Berzaghi, P. Use of a near infrared portable instrument in monitoring anaerobic digestion process parameters and nutrients in digestates. Proceeding of Biogas Science Conference 2018, Lingotto Conference Center, Torino, Italy, 17-19 September 2018; Biogas Science: Tulln an der Donau, Austrian, 2018.

64. Dolud, M.; Andree, H.; Hügle, T. Rapid analysis of liquid hog manure using near-infrared spectroscopy in flowing condition. In Pecision Livestock Farming '05; Cox, S., Ed.; Wageningen Academic Publishers: Wageningen, The Netherlands, 2005; pp. 115-122.

65. Reeves, J.B.; van Kessel, J.S. Determination of ammonium-N, moisture, total C and total N in dairy manures using a near infrared fibre-optic spectrometer. J. Near Infrared Spectrosc. 2000, 8, 151-160. [CrossRef]

66. Sørensen, P.; Birkmose, T.; Hansen, M.; Sorensen, L. Rapid analysis of animal slurry by NIR spectroscopy. In NJF-Seminar-Manure-an Agronomic and Environmental Challenge; Nordiska Jordbruksforskares Förening (NJF): Uppsala, Sweden, 2005; pp. 95-96. 
67. Williams, P.; Eising, E.; Malley, D.F. Industrial-scale continuous on-line analysis of liquid hog manure by NIRS. NIR News 2020, 31, 25-29. [CrossRef]

68. Xing, L.; Chen, L.J.; Han, L.J. Rapid analysis of layer manure using near-infrared reflectance spectroscopy. Poult. Sci. 2008, 87, 1281-1286. [CrossRef]

69. Zimmermann, A.; Thiessen, E.; Andree, H.; Hartung, E. Einsatz von. Nahinfrarotspektroskopie für eine nährstoffgesteuerte Gülleausbringung In Proceeding of Unternehmens-IT: Führungsinstrument oder Verwaltungsbürde? Referate der 28. GIL Jahrestagung, Kiel, Germany, 10-11 March 2008; Müller, R.A.E., Sundermeier, H.-H., Theuvsen, L., Schütze, S., Morgenstern, M., Eds.; GIL: Esslingen, Germany, 2008; pp. 169-172. ISBN 978-3-88579-219-2.

70. Beć, K.B.; Grabska, J.; Siesler, H.W.; Huck, C.W. Handheld near-infrared spectrometers: Where are we heading? NIR News 2020, 31, 28-35. [CrossRef]

71. Zimmermann, A.; Hartung, E. Abschlußbericht zum BLE-Projekt: Nährstoffgesteuerte Gülleausbringung: -05UM003. 2009. Available online: https:/ / docplayer.org/14127726-Abschlussbericht-zum-ble-projekt-naehrstoffgesteuerte-guelleausbringung05um003.html (accessed on 22 January 2022).

72. Drücker, H. Organische Düngung. Jahrb. Agrartech. 2019, 31, 31. [CrossRef]

73. Hunger, R. Dank Online Sensoren Licht ins Dunkel bringen Nährstoffgehalte in der Gülle bestimmen: Ergänzung zum Schwerpunkt «Hofdünger»; Schweizer Landtechnik: Riniken, Switzerland, 2021.

74. Hoffmann, S.; Saev, M.; Wehlert, N. TENIRS. In Fallstudien zur Gründung und Entwicklung innovationsorientierter Unternehmen; Walter, A., Rasmus, A., Riesenhuber, F., Schmidthals, J., Dickel, P., Eds.; Springer: Wiesbaden, Germany, 2014; pp. 173-188. ISBN 978-3-658-03597-6.

75. Severin, K.; Hoffmann, A.; Lichti, F.; Olfs, H.-W.; Rest, T.; Tillmann, P. Die Nahinfrarotspektroskopie (NIRS) zur Untersuchung von Güllen und Gärresten; Standpunkte des VDLUFA.; Verband Deutscher Landwirtschaftlicher Untersuchungs und Forschungsanstalten e.V.: Speyer, Germany, 2019.

76. Chen, L.; Xing, L.; Han, L. Review of the application of near-infrared spectroscopy technology to determine the chemical composition of animal manure. J. Environ. Qual. 2013, 42, 1015-1028. [CrossRef] 